\title{
Differential Vesicular Targeting and Time Course of Synaptic Secretion of the Mammalian Neurotrophins
}

\author{
Tanja Brigadski, ${ }^{1}$ Matthias Hartmann, ${ }^{1,2}$ and Volkmar Lessmann ${ }^{1}$ \\ ${ }^{1}$ Institute of Physiology and Pathophysiology, Johannes Gutenberg-University, 55128 Mainz, Germany, and ${ }^{2}$ Department of Molecular Neurobiochemistry, \\ Ruhr-University, 44780 Bochum, Germany
}

Neurotrophins are a family of secreted neuronal survival and plasticity factors comprising NGF, BDNF, neurotrophin-3 (NT-3), and NT-4. Whereas synaptic secretion of BDNF has been described, the routes of intracellular targeting and secretion of NGF, NT-3, and NT-4 in neurons are poorly understood.

To allow for a direct comparison of intracellular targeting and release properties, all four mammalian neurotrophins were expressed as green fluorescent protein fusion proteins in cultured rat hippocampal neurons. We show that BDNF and NT-3 are targeted more efficiently to dendritic secretory granules of the regulated pathway of secretion (BDNF, in $98 \%$ of cells; NT-3, 85\%) than NGF (46\%) and NT-4 (23\%). For all NTs, the remaining cells showed targeting to the constitutive secretory pathway. Fusing the BDNF pre-pro sequence to NT-4 directed NT-4 more efficiently to the regulated pathway of secretion.

All neurotrophins, once directed to the regulated secretion pathway, were detected near synapsin I-positive presynaptic terminals and colocalized with PSD-95-DsRed (postsynaptic density-95-Discosoma red), suggesting postsynaptic targeting of the neurotrophins to glutamatergic synapses. Depolarization-induced release of all neurotrophins from synaptic secretory granules was slow (delay in onset, $10-30 \mathrm{~s} ; \tau=120-307 \mathrm{~s}$ ) compared with transmitter release kinetics monitored with FM4-64 [ $N$-(3-triethylammoniumpropyl)-4-(6-(4diethylamino)phenyl)hexatrienyl)pyridinium dibromide] destaining (onset, $<5 \mathrm{~s} ; \tau=13 \pm 2 \mathrm{~s}$ ). Among the neurotrophins, NT-4 secretion was most rapid but still proceeded 10 times more slowly than transmitter secretion. Preincubation of neurons with monensin (neutralizing intragranular $\mathrm{pH}$, thus solubilizing the peptide core) increased the speed of secretion of BDNF, NGF, and NT-3 to the value of NT-4. These data suggest that peptide core dissolution in secretory granules is the critical determinant of the speed of synaptic secretion of all mammalian NTs and that the speed of release is not compatible with fast transmitter-like actions of neurotrophins.

Key words: NGF; NT-3; NT-4; BDNF; release; PSD-95; VAMP; FM4-64; peptide; secretion; neurotrophins

\section{Introduction}

The mammalian neurotrophins (NTs) NGF, BDNF, NT-3, and NT-4 were discovered initially based on their impact on the survival and differentiation of developing neurons. More recently, accumulating evidence suggested an additional role of neurotrophins in synaptic plasticity (Poo, 2001; Lu, 2003). In comparison to the wealth of knowledge regarding the biological downstream targets of neurotrophin action, our knowledge regarding the sites and mechanisms of neurotrophin secretion is very limited (Lessmann et al., 2003). The location and the time course of neurotrophin secretion, however, are critical determinants of the role NTs can play in synaptic plasticity.

As determined by ELISA measurements, endogenous BDNF is

Received July 23, 2004; revised June 24, 2005; accepted June 29, 2005.

This work was funded by grants from the Deutsche Forschungsgemeinschaft to V.L. (SFB 509 and SFB 553). We thank R. A. Murphy, R. Heumann, and P. Wahle for kindly providing hNT-3, mNGF, and hNT-4 expression plasmids, D. Bredt for generously providing PSD-95-GFP, W. Huttner and L. DeGennaro for the kind gift of anti-Sgll and anti-synapsin antibodies, Sabine Rickheim-Lowack for excellent technical assistance, and Heiko Luhmann for generous support.

Correspondence should be addressed to Dr. Volkmar Lessmann, Institute of Physiology, Johannes GutenbergUniversity, Duesbergweg 6/Room 03-623, 55128 Mainz, Germany. E-mail: lessmann@uni-mainz.de. DOI:10.1523/JNEUROSCI.1776-05.2005

Copyright $\odot 2005$ Society for Neuroscience $\quad$ 0270-6474/05/257601-14\$15.00/0 secreted in response to patterned high-frequency electrical stimulation of cultured neurons (Balkowiec and Katz, 2000, 2002; Lever et al., 2001). Likewise, overexpressed NGF and BDNF have been shown to be released from transfected neurons during high$\mathrm{K}^{+}$-induced depolarization (Blochl and Thoenen, 1995; Goodman et al., 1996). Most recently, using overexpressed green fluorescent protein (GFP)-tagged BDNF, we and others were able to resolve activity-dependent synaptic secretion of BDNF at glutamatergic synapses (Hartmann et al., 2001; Kojima et al., 2001).

A spatially and time-resolved analysis of the release of NGF, NT-3, and NT-4, however, was lacking. Importantly, it is unknown whether secretion of these NTs takes place at synapses. Given the fundamental implications of NT secretion in human memory formation and neurodegenerative diseases (Egan et al., 2003; Gauthier et al., 2004; Heese et al., 2004), understanding the secretion process of NTs seems extremely important.

BDNF has been identified unambiguously to be localized in secretory granules of the regulated pathway of secretion (Haubensak et al., 1998). However, the routes of intracellular targeting of the other neurotrophins are discussed controversially. Overexpressed NGF and NT-3 have been described to be either targeted to secretory granules (Blochl and Thoenen, 1996; $\mathrm{Wu}$ et al., 2004) or were found in the constitutive pathway of 
secretion (Mowla et al., 1999; Farhadi et al., 2000). For NT-4, targeting to only the constitutive pathway was suggested by one previous study (Hibbert et al., 2003). These disparate findings now prompted us to evaluate neuronal targeting and synaptic secretion of the four mammalian neurotrophins under identical conditions. We overexpressed GFP-tagged NGF, BDNF, NT-3, and NT-4 in hippocampal neurons and explored their vesicular targeting and synaptic release properties. Hippocampal neurons were chosen, because all NTs have been described to be expressed endogenously in this brain area (for review, see Lessmann et al., 2003).

Our results demonstrate that all NTs can be routed to postsynaptic granules of the regulated pathway of secretion, albeit with different efficiencies: BDNF and NT-3 are released from granules in response to synaptic depolarization in $>80 \%$ of neurons, whereas NGF and NT-4 show markedly less synaptic targeting ( $<50 \%)$. For all NTs, the remaining cells displayed targeting to the constitutive pathway of secretion. Comparison with $\mathrm{N}$-(3-triethylammoniumpropyl)-4-(6-(4-diethylamino)phenyl)hexatrienyl)pyridinium dibromide (FM4-64) destaining of presynaptic transmitter vesicles revealed that NT secretion proceeds 10 times more slowly than conventional transmitter release, arguing strongly against a role of NTs as fast transmitters in hippocampal neurons (cf. Kafitz et al., 1999).

\section{Materials and Methods}

Construction of neurotrophin-GFP expression vectors. Rat pre-proBDNF-GFP (BDNF-GFP) had been constructed as described previously (Haubensak et al., 1998). Mouse pre-pro-NGF-GFP (NGF-GFP) was constructed by PCR methods from a mouse pre-pro-NGF-containing pcDNA3 vector coding for the short version of the NGF precursor (Edwards et al., 1988). PCR primers were designed to introduce an EcoRI cleavage site at the $5^{\prime}$ end and a BamH1 restriction site at the $3^{\prime}$ end, respectively. In addition, the $3^{\prime}$ primer deleted the NGF stop codon, removed the last two amino acids (RG) of the coding sequence, and mutated the remaining final arginine to alanine to avoid cleavage at these dibasic residues by protein convertases, thus hindering separation of GFP from NGF by posttranslational modifications. The PCR product was digested by EcoRI and BamH1 and ligated into the enhanced GFP-N1 (EGFP-N1) vector (Clontech, Cambridge, UK). In this construct, a seven amino acid spacer is introduced between the NGF and the EGFP portion of the fusion protein. Human pre-pro-NT-3-GFP and pre-proNT-4-GFP were generated from pre-pro-NT-3 in pBluescript (kindly provided by R. A. Murphy, Salk Institute for Biological Studies, San Diego, CA) and pre-pro-NT-4 in pCMX (kindly provided by P. Wahle, Ruhr-University, Bochum, Germany) using a similar PCR-based cloning strategy as described above for NGF but without changing C-terminal sequences. Absence of PCR-introduced mutations were confirmed by DNA sequencing of all constructs.

Cell culture. COS7 and pheochromocytoma 12 (PC12) cells were grown at $37^{\circ} \mathrm{C}$ in $5 \% \mathrm{CO}_{2}$ in DMEM (Invitrogen, San Diego, CA), supplemented with $5 \mathrm{~mm}$ glucose, $1 \mathrm{~mm}$ Glutamax (Invitrogen), $10 \mathrm{~mm}$ HEPES, $37.5 \mu \mathrm{g} / \mathrm{ml}$ insulin, $25 \mathrm{U} / \mathrm{ml}$ penicillin, and $25 \mu \mathrm{g} / \mathrm{ml}$ streptomycin and containing either $10 \%$ fetal calf serum (COS7 cells) or $5 \%$ horse serum and $10 \%$ fetal calf serum (PC12 cells). After reaching confluence, cells were passaged 1:5 (PC12) or 1:10 (COS7). For transfection, cells were plated in uncoated cell culture dishes $(6 \mathrm{~cm})$ at a density of 40,000 cells $/ \mathrm{cm}^{2}$ (COS7) or on polyornithine-coated $[1 \mathrm{mg} / \mathrm{ml}$ in borate buffer, $4 \mathrm{~h}$ at room temperature (RT)] coverslips at a density of $70,000 \mathrm{cells} / \mathrm{cm}^{2}$ (PC12). Transfection was performed $1 \mathrm{~d}$ after plating. Supernatants of NT-GFP-expressing COS7 cells for the PC12 cell fiber outgrowth assay and the Western blot experiments were collected $24-48 \mathrm{~h}$ after transfection at dilutions as indicated.

Hippocampal microcultures. Dissociated postnatal rat hippocampal microcultures were prepared as described previously (Lessmann and Heumann, 1998) with minor modifications: primary postnatal [postna- tal day 0 (P0) to $\mathrm{P} 2$ ] neocortical astrocytes were isolated and cultured for 2-4 weeks in DMEM medium containing 10\% FCS until expanded to confluence. Astrocytes were passaged and seeded on glass coverslips at a density of 80,000 cells per $3.5 \mathrm{~cm}$ dish in DMEM/10\% FCS to yield astrocyte islands of $100-300 \mu \mathrm{m}$ in diameter after $7-14 \mathrm{~d}$ in vitro (DIV). Postnatal rat ( $\mathrm{P} 0-\mathrm{P} 2)$ hippocampal neurons were plated in DMEM/10\% FCS at a density of 1-10 neurons per astrocyte island onto the astrocyte coverslips. After $20 \mathrm{~h}$, the plating medium was exchanged to serum-free medium (Neurobasal with 2\% B27 supplement; Invitrogen).

Transfection. Rat hippocampal microcultures were transfected with the respective expression plasmids at 8-9 DIV using the $\mathrm{Ca}^{2+}$ phosphate precipitation method as described previously (Haubensak et al., 1998). During incubation ( $3 \mathrm{~h}$ ), $10 \mu \mathrm{M}$ DNQX and $100 \mu \mathrm{M}$ D,L-APV were added. Cells were used for experiments $1-3 \mathrm{~d}$ after transfection (9-11 DIV).

COS7 cells were transfected transiently with the NT-GFP constructs using the polyethylenimine (PEI) protocol, as described previously (Boussif et al., 1995; Haubensak et al., 1998). The PEI stock solution contained 0.9 mg of PEI solution (50\%; 800,000 kDa; Fluka, Neu-Ulm, Germany) per $\mathrm{ml}$ of $\mathrm{H}_{2} \mathrm{O}$, pH adjusted to $6.5 \mathrm{using} \mathrm{HCl}$. The transfection solution contained $20 \mu \mathrm{g}$ of plasmid DNA and $60 \mu \mathrm{l}$ of PEI stock per ml of $150 \mathrm{~mm} \mathrm{NaCl}$. Transfections were performed using the equivalent of 5 $\mu \mathrm{g}$ of plasmid DNA in transfection solution per $\mathrm{ml}$ of serum-free DMEM. After $3 \mathrm{~h}$ of incubation at $37^{\circ} \mathrm{C}\left(5 \% \mathrm{CO}_{2}\right)$, cells were washed with normal culture medium (DMEM/10\% FCS) and cultured for 2 more days. The cell culture supernatants of these cells were collected and incubated with the TrkA and TrkB receptor-expressing transfected PC12 cells to determine fiber outgrowth capacity of the GFP-tagged NTs. PC12 cells were transfected with TrkB expression plasmids (Klau et al., 2001) using the same PEI transfection method.

Western blot. Whole-cell lysates and culture medium supernatants of NT-GFP-expressing COS7 cells were analyzed $48 \mathrm{~h}$ after transfection. EGFP-transfected and wild-type (wt) BDNF-transfected COS7 cells (Haubensak et al., 1998) were used as controls. Aliquots of cell lysates and supernatants were subjected to SDS-PAGE and transferred to nitrocellulose membranes $(0.2 \mu \mathrm{m}$; Schleicher \& Schüll BioScience, Dassel, Germany) using standard protocols (Sambrook et al., 1989). After blocking, the nitrocellulose membrane was incubated $(1 \mathrm{~h}, \mathrm{RT})$ either with a rabbit anti-GFP antibody (1:200; Abcam, Cambridge, UK) or with a rabbit anti-BDNF antibody (1:2000; Santa Cruz Biotechnology, Santa Cruz, CA) in Tris-buffered saline, containing $10 \mathrm{~mm}$ Tris/ $\mathrm{HCl}, 150 \mathrm{~mm} \mathrm{NaCl}$, and $0.05 \%(\mathrm{v} / \mathrm{v})$ Tween 20 . After incubation $(1 \mathrm{~h}, \mathrm{RT})$ with secondary antibody (HRP-conjugated anti-rabbit IgG; 1:2000; Sigma, St. Louis, $\mathrm{MO}$ ), binding of secondary antibodies was detected using an ECL detection kit (Amersham Biosciences, Braunschweig, Germany) according to the manufacturer's instruction.

FM4-64 staining. Activity-dependent labeling of presynaptic terminals was performed as described previously (Klau et al., 2001; Mohrmann et al., 2003). Briefly, coverslips with hippocampal neurons were incubated for $1 \mathrm{~min}$ at RT in HEPES-buffered saline (HBS) containing $50 \mathrm{~mm} \mathrm{~K}$ (replacing an equal amount of $\mathrm{Na}^{+}$), $2 \mathrm{mM} \mathrm{Ca}^{2+}, 1 \mathrm{mM} \mathrm{Mg}^{2+}$, and 10 $\mu \mathrm{M}$ FM4-64 (Molecular Probes, Eugene, OR). Cells were washed four times in dye-free $\mathrm{HBS}$ with low $\mathrm{K}^{+}$and without $\mathrm{Ca}^{2+}$ and were transferred into the recording chamber of an inverted microscope.

Cotransfection with synaptic marker proteins. Discosoma red-vesicleassociated membrane protein (DsRed-VAMP) and postsynaptic density-95-DsRed (PSD-95-DsRed) were generated by PCR methods from a rat VAMP cDNA plasmid and a PSD-95-GFP expression plasmid (kindly provided by D. Bredt, University of California, San Francisco, CA) and cloned into the EGFP-C1 (VAMP) or EGFP-N1 vector (PSD95) from Clontech. Intact synaptic targeting of both proteins was confirmed by colocalization of DsRed-VAMP and PSD-95-DsRed with the green fluorescent activity-dependent label of active synapses FM1-43 [N-(3-triethylammoniumpropyl)-4-(4-dibutylamino)styryl)pyridinium dibromide] in cells only expressing these DsRed fusion proteins (our unpublished observation). To identify presynaptic and postsynaptic structures, respectively, in NT-GFP-expressing hippocampal neurons, cells were cotransfected with the respective NT-GFP construct together with either DsRed-VAMP or with PSD-95-DsRed (DNA ratio GFP:DsRed, 1:1). 
Immunocytochemistry. Coverslips of transfected cells were fixed (4\% formaldehyde in PBS) and permeabilized (0.1\% Triton X-100) using standard procedures (Haubensak et al., 1998). Secretogranin antibody (rabbit; 1:200; kindly provided by W. Huttner, Max-Planck-Institute of Molecular Cell Biology and Genetics, Dresden, Germany), synapsin I (SynI) antibody (mouse; 1:400; kindly provided by L. DeGennaro, University of Rome "La Sapienza," Rome, Italy), calnexin antibody (rabbit; 1:200; Stressgen, Victoria, British Columbia, Canada), and GM130 antibody (rabbit; 1:100; BD Biosciences, Franklin Lakes, NJ) were incubated for $1 \mathrm{~h}$ at RT followed by incubation (RT, $1 \mathrm{~h}$ ) with Alexa Red-coupled anti-rabbit secondary antibody or Alexa Red-coupled anti-mouse secondary antibody (both 1:1000; Molecular Probes). Immunocytochemical detection of overexpressed and endogenous neurotrophins was performed using the following polyclonal sheep primary antibodies $(24 \mathrm{~h}$ incubation at RT): anti-BDNF (1:200), anti NT-3 (1:200), anti-NT-4 (1:200), anti-NGF (1:200; all from Chemicon, Temecula, CA). Secondary Alexa Red-coupled anti-sheep antibodies were incubated for $1 \mathrm{~h}$ at RT.

Fluorescence imaging. Visualization of epifluorescence signals was performed as described previously (Hartmann et al., 2001). Briefly, coverslips with transfected cells were transferred into Petriperm dishes (Vivascience-Sartorius, Hannover, Germany) with folio bottom and inspected with an inverted microscope (IX70; Olympus, Melville, NY) using $40 \times$ [numerical aperture (N.A.), 1.0] and $100 \times$ (N.A., 1.35) oil immersion objectives. Image capture was performed using a cooled CCD camera (Sensys 1401E; PhotoMetrics, Huntington Beach, CA) controlled by MetaView software (Universal Imaging Corporation, West Chester, PA). Exposure times were chosen such that saturation was avoided. Processing of images was performed by MetaMorph (Universal Imaging Corporation) and Adobe Photoshop (Adobe Systems, San Jose, CA) software without compromising the evident primary image information. However, in the Figures, fluorescence in the soma and the proximal dendrites is often enhanced close to saturation to make clearly visible also single vesicles in distal neurites.

Confocal imaging. Colocalization of NTs with the different markers was evaluated with a Nipkow spinning disk confocal system (Visitech, Sunderland, UK), attached to a conventional fluorescence microscope (Olympus BX51 WI) equipped with high-aperture oil immersion objectives (40×, N.A., 1.0;100×, N.A., 1.35). Full-frame image capture was performed with a cooled CCD camera (CoolSnap HQ; Roper Scientific, Trenton, NY). Green and red fluorescence was excited with the 488 and the $568 \mathrm{~nm}$ lines of a krypton/argon laser (Laser Physics, West Jordan, UT).

Quantification of DsRed and NT colocalization in neurites. Hippocampal neurons were cotransfected with DsRed and the respective GFPtagged NT. Two days after transfection, the red fluorescent picture was taken as a measure of neurite area, and the green fluorescent picture was taken as a measure of neurite area occupied by NT granules. Both photographs for each neuron were thresholded by eye and converted to binary pictures. The soma area (including the principle dendrite; diameter, $>3 \mu \mathrm{m}$ ) was deleted, and the percentage of overlap between the two binary pictures in all neurites was calculated and is given as percentage of total neurite area. All calculations were performed with MetaMorph software.

Neurotrophin release. Real-time imaging of synaptic secretion of GFPtagged NTs was performed as described previously (Hartmann et al., 2001). Coverslips of NT-GFP-expressing hippocampal neurons were mounted in the perfusion chamber of an inverted fluorescence microscope. Where indicated, synaptic localization of NT-GFP vesicle clusters was visualized using FM4-64 staining or cotransfection with DsRedVAMP or PSD-95-DsRed. Cells with synaptically localized NT-GFP were superfused locally with HBS throughout the experiment, and images were captured at 5 or 10 s intervals, respectively. After 5 min control period, cells were depolarized for $5 \mathrm{~min}$ by superfusing $50 \mathrm{~mm} \mathrm{~K}{ }^{+}$containing HBS at RT. Regions containing synaptic clusters of NT-GFP were analyzed over time for changes in intracellular fluorescence intensity. Background fluorescence levels of a void region in the same field of view was recorded in parallel and subtracted. Fluorescence decrease caused by photobleaching was corrected for by monoexponential extrapolation of the fluorescence decrease during an initial 5 min control period over the whole recording time. Original fluorescence values were divided by the respective value of this bleaching curve, thus providing a measure of the fluorescence decrease caused by release. This fluorescence decrease has been shown previously to reflect release of NTs (Hartmann et al., 2001). In experiments with monensin, photobleaching during monensin application was extrapolated and compensated as described above.

Constitutive secretion of neurotrophins was measured in the presence of $1 \mu \mathrm{M}$ TTX and in nominal $\mathrm{Ca}^{2+}$-free HBS to exclude observation of spontaneous regulated secretion. Exposure times and the intensity of excitation light were minimized to eliminate photobleaching. Changes in fluorescence intensity were recorded during $10 \mathrm{~min}$ of continuous superfusion with the TTX-containing $\mathrm{Ca}^{2+}$ free HBS. The amount of constitutively secreted NT was determined as the percentage decrease of fluorescence intensity during the $10 \mathrm{~min}$ of observation. At the end of the recording, $50 \mathrm{~mm} \mathrm{KCl}$ in normal $\mathrm{HBS}$ were used to test for regulated secretion in the same cell.

PC12 fiber outgrowth assay. PC12 cells express the TrkA tyrosine kinase receptor for NGF and show fiber outgrowth in response to activation of Trk signaling (Tischler et al., 1978). To also test for TrkB liganddependent fiber outgrowth, PC12 cells plated on glass coverslips were cotransfected with TrkB.FL and GFP (wt TrkB:GFP, 3:1) (Klau et al., 2001). One day after transfection, recombinant human (rh) BDNF, NGF, or NT-4 (all from Alamone Labs, Jerusalem, Israel), respectively, was added at $100 \mathrm{ng} / \mathrm{ml}$. Alternatively, supernatants from COS cells expressing the different NT-GFPs were added at dilutions as indicated. Four days later, the percentage of transfected cells (identified by coexpression of GFP) with fibers longer than their respective soma diameter was determined.

Reagents. Recombinant human NTs were purchased from Alamone Labs. Monensin was obtained from Sigma.

\section{Results}

GFP-tagged neurotrophins are processed correctly and retain biological activity

To follow time-resolved synaptic secretion of all mammalian neurotrophins, we generated GFP fusion constructs of BDNF (Haubensak et al., 1998), NGF, NT-3, and NT-4, respectively (see Materials and Methods). After expression in COS7 cells, the respective pro-NTs were detected in anti-GFP Western blots of COS cell lysates (Fig. 1A). Two days after transfection, COS cell supernatants were found to contain predominantly the processed and subsequently released mature forms of all four neurotrophins. The detected molecular weights (MWs) of the respective pro-NTs and their mature counterparts corresponded well with the expected sizes of the generated fusion proteins (mNGF-GFP precursor, $57 \mathrm{kDa}$; mature mNGF-GFP, 43 kDa; hNT-4-GFP, 53 $\mathrm{kDa}$ (precursor) and $44 \mathrm{kDa}$ (mature); hNT-3-GFP, $59 \mathrm{kDa}$ (precursor) and $43 \mathrm{kDa}$ (mature); rBDNF-GFP, $58 \mathrm{kDa}$ (precursor) and $43 \mathrm{kDa}$ (mature); MW of GFP tag alone, $29 \mathrm{kDa}$ ). Biological activity of the secreted mature NTs in COS cell supernatants was determined by a fiber outgrowth assay in PC12 cells (see Materials and Methods). In PC12 cells overexpressing the TrkB receptor, the TrkB ligands BDNF-GFP and NT-4-GFP derived from COS cell supernatants induced fiber outgrowth to a similar extent as $100 \mathrm{ng} / \mathrm{ml}$ recombinant BDNF and NT-4, respectively. Likewise, COS cell-derived NGF-GFP supported PC12 cell fiber outgrowth via the endogenously expressed TrkA NGF receptor (all GFP-tagged NTs significantly different from EGFP control with $p<0.01$; all supernatants not significantly different from the respective recombinant factors with $p>0.1$ ) (Fig. $1 B, C$ ). In these experiments, NT-3-GFP (which at higher concentrations is known to activate TrkA and TrkB receptors) also induced fiber outgrowth, although this effect did not reach significant levels. Together, these results suggest that all GFP-tagged NTs are efficiently processed by the secretory machinery of mammalian cells and can effectively bind and activate the Trk kinase receptors. Thus, the phys- 
iological function of the NTs seems to remain primarily unaffected by the GFP extensions.

To further quantify the relative potency of BDNF-GFP versus wt BDNF to activate TrkB, equal amounts of BDNF-GFP and wt BDNF, respectively, were tested in the outgrowth assay: supernatants of COS cells overexpressing BDNF-GFP and wt BDNF, respectively, were found to contain comparable amounts of BDNF, as estimated by an anti-BDNF Western blot (Fig. $1 D)$. As is evident from Fig. $1 E$, serial dilutions of BDNF-GFP and wt BDNF revealed similar potencies to induce fiber outgrowth, suggesting comparable affinities of wt BDNF and BDNF-GFP in binding and activating $\mathrm{TrkB}$ receptors.

Expression pattern of neurotrophins in hippocampal neurons

Hippocampal neurons are known to endogenously express all four mammalian NTs and thus are supposed to have the capacity for processing, targeting, and secretion of these proteins (Korsching et al., 1985; Ernfors et al., 1990, 1992; Hofer et al., 1990; Maisonpierre et al., 1990; Zhou and Rush, 1994; Friedman et al., 1998; Lessmann et al., 2003). To enable a direct comparison of the subcellular targeting of the different NTs under identical conditions, we expressed the four GFP-tagged NTs in sister cultures of rat hippocampal neurons. Two days after transfection (i.e., at 10 DIV), subcellular targeting was visualized in living neurons by epifluorescence microscopy (Fig. 2). One-third of the transfected neurons (regardless of the NT under investigation) displayed homogenous bright fluorescence throughout the entire cell, including all processes and the nucleus (data not shown). These cells were excluded from analysis because of lack of any visible targeting. Only cells exhibiting a patchy pattern of intracellular GFP fluorescence, which excluded the nucleus, were further analyzed. These neurons could be subdivided into two groups: (1) distal expressers, showing vesicular targeting to distal neurites [i.e., $>30 \mu \mathrm{m}$ apart from the soma (compare Fig. 2)] or (2) proximal expressers, showing retention of fluorescent punctas primarily in the soma, thus sparing distal neurites but showing diffuse GFP signals in the dendrites (Fig. 2A). Whereas BDNFGFP and NT-3-GFP displayed distal vesicular targeting to the dendrites in the majority of neurons (BDNF-GFP, $98 \pm 2 \%$; NT-3-GFP, $85 \pm 8 \%$ ), distal vesicular targeting of NGF-GFP and NT-4-GFP was observed less frequently (NGF, $43 \pm 10 \%$; NT-4, $26 \pm 6 \%$; both significantly different from BDNF-GFP and NT$3-$ GFP with $p<0.01$ ). Because distal vesicular and proximal expression with faint dendritic GFP signal, respectively, were virtually exclusive phenomena for any given cell, the classification
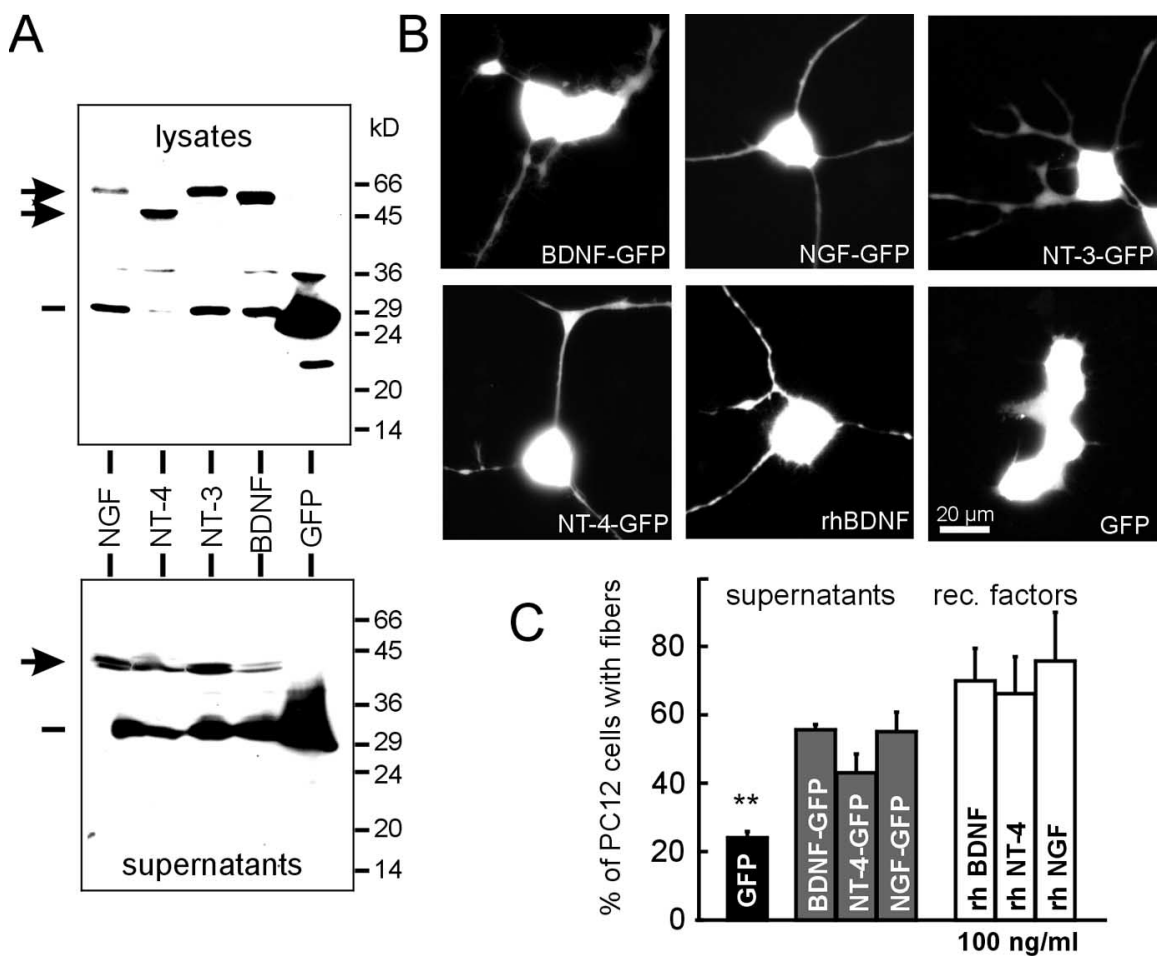

E

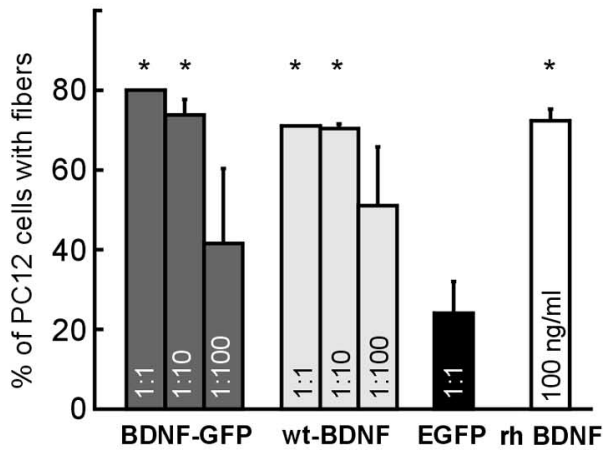

Figure 1. Intact processing and biological function of GFP-tagged neurotrophins. GFP-tagged NTs were expressed in COS7 cells, and cell lysates and supernatants were analyzed $2 \mathrm{~d}$ after transfection. $\boldsymbol{A}$, Anti-GFP Western blot reveals dominant presence of the NT precursors in the cell lysates (top, arrows, NT-4 precursor with lower MW than the other NTs) and of the correctly processed mature NTs in the COS cell supernatants (bottom, arrow). The horizontal marks at the left indicate the position of GFP. $B$, NT-induced fiber outgrowth of PC12 cells via overexpressed TrkB and endogenously expressed TrkA receptors, respectively. PC12 cells were cotransfected at 1 DIV with TrkB and GFP plasmids and were incubated for $3 \mathrm{~d}$ with supernatants from COS cells overexpressing (for 2 d) GFP-tagged BDNF, NGF, NT-3, NT-4, or wt GFP, respectively, or were incubated with rhBDNF. C, Quantification of NT-induced fiber outgrowth of $\mathrm{PC} 12$ cells after $3 \mathrm{~d}$ of incubation with $\mathrm{COS}$ cell supernatants and recombinant (rec.) neurotrophins. ${ }^{* *}$ Significantly different from all NTs with $p<0.01$. D, Anti-BDNF Western blot of COS cells expressing wt BDNF GFP in the supernatants used in $\boldsymbol{E}$. $\boldsymbol{E}, \mathrm{PC1} 2$ cell fiber outgrowth assay of supernatants and recombinant BDNF analyzed in $\boldsymbol{D}$. * Significantly different from EGFP with $p<0.01$. Data in $\boldsymbol{C}$ and $\boldsymbol{E}$ are from $>100$ cells per experiment and from more than two independent preparations for each condition. Error bars represent $\mathrm{SES}$.

into these two separate groups matches the observed pattern of expression more closely than calculating the average dendritic colocalization of vesicles over all cells. The existence of these two groups was substantiated by experiments in which we coexpressed BDNF-GFP or NT-4-GFP with soluble DsRed and quantified the percentage of dendritic area (indicated by the DsRed fluorescence) that was covered by NT vesicles (Fig. 2C). Both NTs clearly showed two distinguishable groups in this analysis. BDNF-GFP neurons with distal vesicular targeting showed $16.5 \pm 1.2 \%$ (NT4-GFP, $12.5 \pm 0.7 \%$ ) colocalization with DsRed (range, 10.6-21.0\%), compared with $3.3 \pm 0.5 \%$ (NT-4- 
A
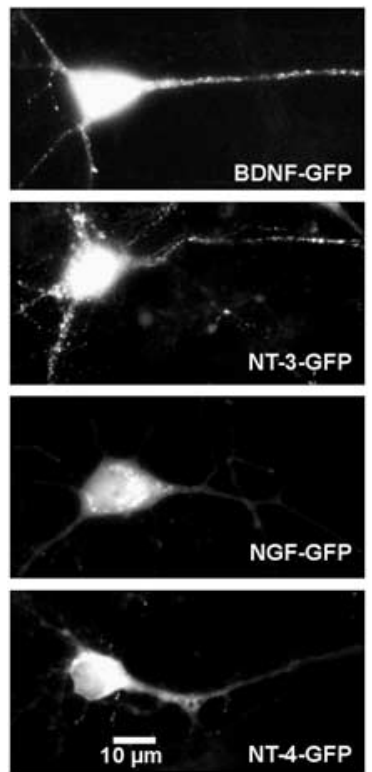

B

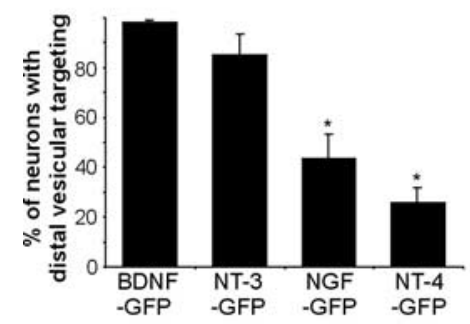

C
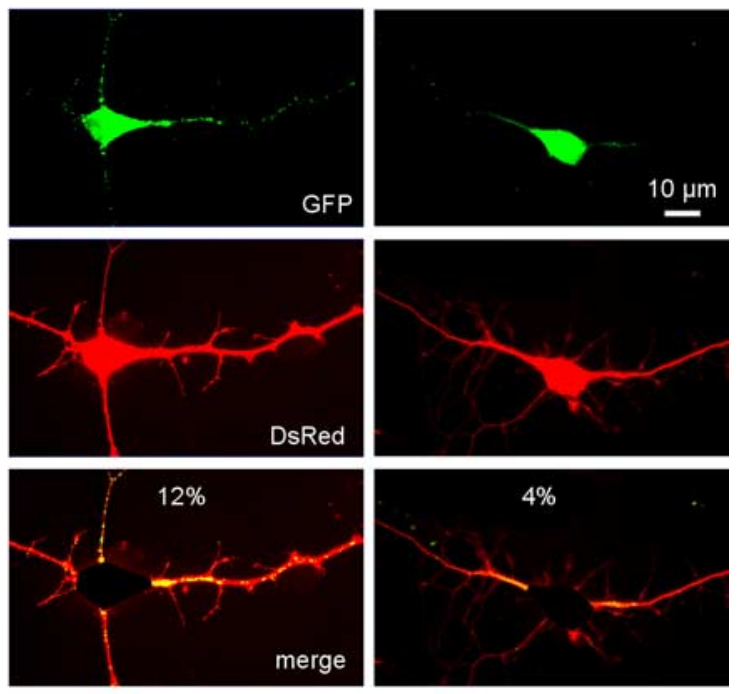

staining with an antibody against the GABA neuron marker GAD67 revealed no correlation between either distal vesicular or proximal expression pattern and GABA expression.

To investigate whether the GFP tag influences the targeting pattern, we performed experiments in which untagged versions of all NTs were expressed together with wild-type GFP to identify transfected neurons. In these cells, immunocytochemical detection again revealed preferential distal vesicular targeting for BDNF and NT-3 compared with NGF and NT-4 (see supplemental Fig. 1, available at www.jneurosci.org as supplemental material). Analysis of distal versus proximal expressers for BDNF and NT-4 disclosed a quantitative difference as observed with the GFP-tagged counterparts.

Using the same antibodies, we also attempted to visualize endogenously expressed NTs in our hippocampal neurons. Whereas the abundantly expressed BDNF could be detected previously as punctate staining in dendrites of many neurons (cf. Haubensak et al., 1998; Swanwick et al., 2004), the more seldom expressed NT-3, NT-4, and NGF were only occasionally observed in our hippocampal cultures. As a result of the low signal-to-noise ratio in dendrites, proximal expressers could not be securely distinguished from cells showing unspecific background fluorescence, whereas distal vesicular expressers could be clearly identified (see supplemental Fig. 2, available at www.jneurosci.org as supplemental material). Thus, quantitative analysis of distal versus proximal targeting of endogenously expressed NTs was not feasible.

Importantly, these results with untagged NTs and with the endogenously expressed NTs clearly reveal that all NTs show, at least to some extent, distal vesicular targeting, which thus is a physiological sorting pattern for all NTs in hippocampal neurons.

Because our results suggested a cellspecific differential sorting of NTs, we argued that in a clonal rat neuronal cell line, cell-specific variations in targeting should be negligible. We therefore expressed all GFP-tagged NTs in the rat PC12 cell line,

GFP, $3.2 \pm 0.6 \%$; range, $1.0-5.4 \%$ ) overlap with DsRed for neurons showing proximal expression of BDNF-GFP or NT-4-GFP. The difference in dendritic localization for distal versus proximal expressers was highly significant $\left(p<10^{-7} ; n=29\right.$ cells). Similar results were observed for NT-3 and NGF (data not shown).

Together, these results suggest that a large subpopulation of neurons lack the capacity to sort NGF and NT-4 vesicles into distal dendrites, whereas the vast majority of BDNF and NT-3 expressers show the distal vesicular targeting. However, the distal and the proximal expression pattern, respectively, can be observed for all NTs (compare Fig. 2D). Immunocytochemical which was differentiated into chromaffin-like neurons by addition of NGF (100 ng/ml for $7 \mathrm{~d}$ ) (Tischler and Greene, 1978). Under these conditions, all NTs sorted with equally high efficiency $(70-90 \%)$ into vesicles that were targeted to distal processes (compare supplemental Fig. $3 A$, available at www.jneurosci. org as supplemental material). Thus, PC12 cells behave for all NTs like the distal expressers observed in our hippocampal neurons. Interestingly, undifferentiated PC12 cells (known to target NTs to the constitutive pathway, because the regulated pathway is not well developed under these conditions) (Nomoto et al., 2000) showed 
diffuse expression of all GFP-tagged NTs throughout the cells (see supplemental Fig. $3 B$, available at www.jneurosci.org as supplemental material), resembling the diffuse GFP signal in proximal expressers. This corroborates the notion that the observed sorting of a NT is ruled by the individual targeting capacity of a given cell and that this capacity can change (e.g., with cell differentiation).

\section{Neurotrophins partition between endoplasmic reticulum, Golgi, and secretory granules}

We further characterized distal and proximal expression patterns by immunocytochemical labeling of the neurons with endoplasmic reticulum (ER) and Golgi markers. As an ER marker, we chose an antibody directed against the ER transmembrane chaperone calnexin, which assists in folding of glycosylated proteins (such as NTs) destined for the Golgi and which has been shown previously to stain rough as well as smooth ER in soma and dendrites of hippocampal neurons (Krijnse-Locker et al., 1995; Weclewicz et al., 1998; Horton and Ehlers, 2003). Cells that displayed the proximal expression pattern showed clear colocalization of calnexin and the respective NT in the soma and additional colocalization of the diffuse NT signals in dendrites with the calnexin staining (Fig. $3 E, F$ ). Whereas the distal expressers showed a similar colocalization with the ER marker in the soma, the distal NT vesicles were spared from calnexin immunoreactivity. Diffuse NT signals that colocalize with calnexin in proximal expressers were essentially absent in distal expressers (Fig. 3A-D). These data suggest that, in dendrites, proximal expressers at least in part retain the expressed NT in ER-derived structures, whereas in distal expressers, a post-ER compartment is reached in the processes.

Proteins destined for the regulated pathway of secretion are known to pass the Golgi compartment and accumulate in secretory granules (Halban and Irminger, 1994). Therefore, we stained our cells with an antibody directed against the Golgi marker GM130, which had been shown previously to partially colocalize with secreted proteins in hippocampal neurons (Horton and Ehlers, 2003). Interestingly, GM130 showed partial colocalization with GFP-tagged NTs in the soma of proximal and distal expressers (Fig. 4). In the dendrites, very few GM130-positive vesicles were stained, which only in distal expressers seldom colocalized with NT vesicles. Importantly, the frequency of GM130positive vesicles in distal and proximal expressers was the same. These results suggest that the GM130-positive fraction of the Golgi compartment is concentrated in the soma and is reached by the NTs in both types of expressers.

Given that most of the NT-GFP-labeled structures in the dendrites of distal vesicular expressers were colocalized neither with the ER marker calnexin nor with the Golgi marker GM130, the question of which cellular compartment was occupied by GFP- labeled NTs in dendrites of these cells arose. As shown by immunofluorescent staining with an antibody directed against the secretory granule marker secretogranin II (SgII) (Huttner et al., 1991; Halban and Irminger, 1994; Ozawa and Takata, 1995), the distal vesicular phenotype corresponded to a targeting of the respective NTs to vesicles of the regulated pathway of secretion (Fig. 5E-I). Interestingly, SgII and NTs colocalized in the soma of distal as well as of proximal expressers, indicating that SgII vesicles are reached in both groups of cells. The often proximal expression pattern of NGF and NT-4 thus reflects less efficient targeting of NGF- and NT-4-containing secretory granules to distal dendrites, compared with BDNF and NT-3.

In an attempt to explore the contribution of pre-pro sequences in directing a NT to distal dendrites, we developed a cDNA construct in which we fused the pre-pro domain of BDNF to the mature portion of NT-4. Interestingly, the pre-pro domain of BDNF targeted NT-4 more efficiently to the regulated pathway than observed for wt NT-4 (pre-pro-BDNF-NT-4-GFP significantly different from NT-4 with $p<0.05$ ) (Fig. 6) and nearly as efficient as BDNF-GFP and NT-3-GFP. However, the pre-pro domain of BDNF alone fused to GFP was not sufficient to reach the regulated pathway (Fig. $6 B$ ). These data indicate that the 
GFP
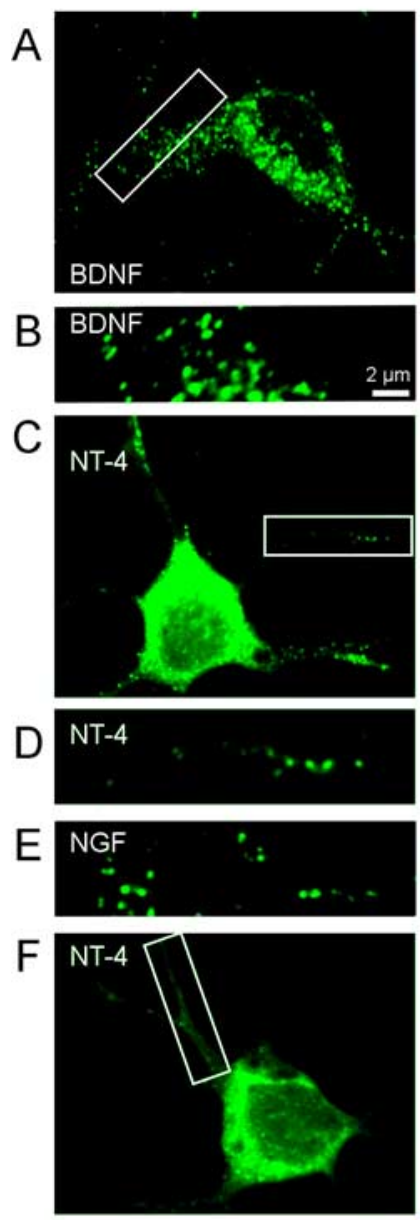

G

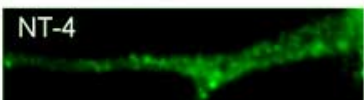

GM130
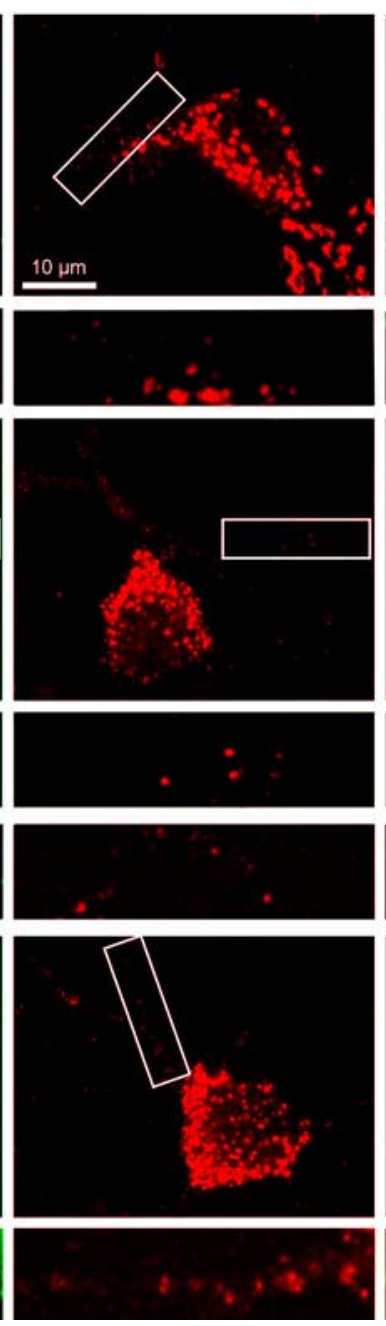

merge
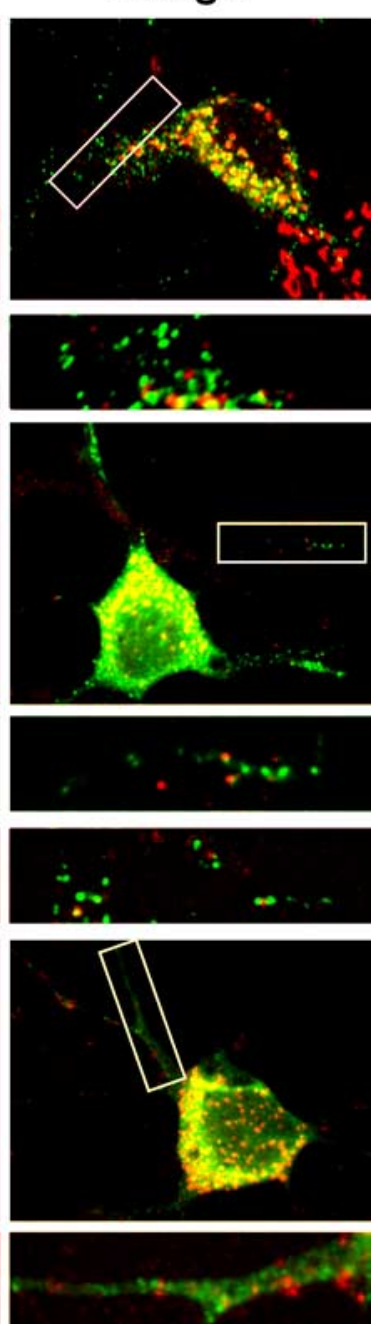

Figure 4. Colocalization of neurotrophins with the Golgi marker GM130. Hippocampal neurons as in Figure 3, immunostained with an antibody directed against the Golgi marker GM130 at 10 DIV. $A-G$ show confocal images of GFP (green) and GM130 (red) fluorescence. $\boldsymbol{A}, \boldsymbol{B}$, BDNF-GFP-expressing cell showing distal vesicular expression (98\% of BDNF cells). C, D, NT-4-expressing neuron displaying distal vesicular localization ( $23 \%$ of NT-4 cells). E, NGF-GFP in a distal vesicular expresser ( $46 \%$ of NGF cells) at high magnification. $\boldsymbol{F}, \mathbf{G}$, NT-4-expressing neuron showing proximal localization with faint NT signals in the periphery. Note that distal and proximal expressers show pronounced colocalization with the Golgi marker in the soma.

pre-pro domain of BDNF contains important determinants for vesicular targeting but also suggest that additional targeting sequences in the mature portion of NTs are required.

\section{Synaptic targeting of neurotrophins}

In the next series of experiments, we asked whether the dendritic vesicles, which are found in distal expressers for all NTs, are targeted to synapses. We thus performed cotransfection experiments for each of the GFP-tagged NTs with the postsynaptic marker of glutamatergic synapses PSD-95-DsRed. As shown in Figure 7, vesicle clusters of all NTs were found to colocalize with PSD-95-DsRed expressed in the same neuron. Furthermore, dendritic NT vesicle clusters of transfected neurons could be found in the vicinity of presynaptic terminals, as revealed by immunocytochemical labeling with an antibody directed against the general presynaptic marker SynI (Fig. 8). In cultures cotransfected with DsRed-VAMP, a similar apposition of dendritic GFP-tagged NTs and presynaptic VAMP was observed (Fig. 9D). Together, these results suggest postsynaptic targeting of NT- containing secretory vesicles to glutamatergic synapses, for all mammalian NTs. This postsynaptic dendritic targeting is in line with the prominent colocalization of all four GFP-tagged NTs with the dendritic marker microtubule-associated protein 2 (data not shown). Finally, we wanted to determine whether the synaptic locations in the vicinity of NT vesicle clusters represented active synapses. We thus performed activity-dependent live staining of presynaptic terminals with FM4-64 (Cochilla et al., 1999), which revealed adjacent localization of the NT containing secretory granules and the FM4-64-labeled active presynaptic terminals (supplemental Fig. 4, available at www.jneurosci.org as supplemental material).

\section{Secretion of neurotrophins}

Next, we asked whether NGF, NT-3, and NT-4 can be secreted at glutamatergic synapses. Therefore, we coexpressed the GFP tagged NTs with synaptic markers (either PSD-95-DsRed or DsRed-VAMP) and monitored secretion by the decrease in intracellular GFP fluorescence intensity (Hartmann et al., 2001). Synaptically localized NT vesicle clusters were depolarized by application of $50 \mathrm{~mm} \mathrm{~K}^{+}$(replacing an equal amount of $\mathrm{Na}^{+}$) in extracellular solution. As verified by electrophysiological recording, application of this elevated $\mathrm{K}^{+}$solution for 5 min reversibly depolarized the neurons to between -25 and $-30 \mathrm{mV}$ membrane potential (data not shown). Cells were continuously superfused with a laminar stream of extracellular solution throughout the experiment to accomplish fast clearance of released peptide. As is evident from the results presented in Figure 9, each of the NTs was released synaptically in response to this depolarization. The change in fluorescence intensity became apparent within the first 10-30 s after the start of the depolarization. In case of BDNF and NT-3, the fluorescence decrease was often preceded by a brief rise in fluorescence intensity (see Figs. 9D, 11C,D). This initial fluorescence increase has been shown previously to result from reduced quenching and thus increase of GFP fluorescence in the secretory granules after neutralization of intravesicular $\mathrm{pH}$ in the context of fusion pore opening (Han et al., 1999; Barg et al., 2002) (see below). Such a depolarization-induced increase in GFP fluorescence was observed less frequently for NT-4 and NGF.

We also performed time-lapse video recordings of constitutive secretion in our hippocampal neurons. Constitutive secretion was measured as intracellular fluorescence decrease during control conditions $\left(4 \mathrm{mM} \mathrm{K}{ }^{+}, 1 \mu \mathrm{M}\right.$ TTX to block spontaneous action potentials; $0 \mathrm{mM} \mathrm{Ca}^{2+}$ to block $\mathrm{Ca}^{2+}$ influx). Somatic and dendritic areas were selected for observation of constitutive release in NT-3-transfected distal expressers and in NGFtransfected proximal expressers, respectively (Fig. 10). Cells expressing soluble GFP were used as negative controls. During the 

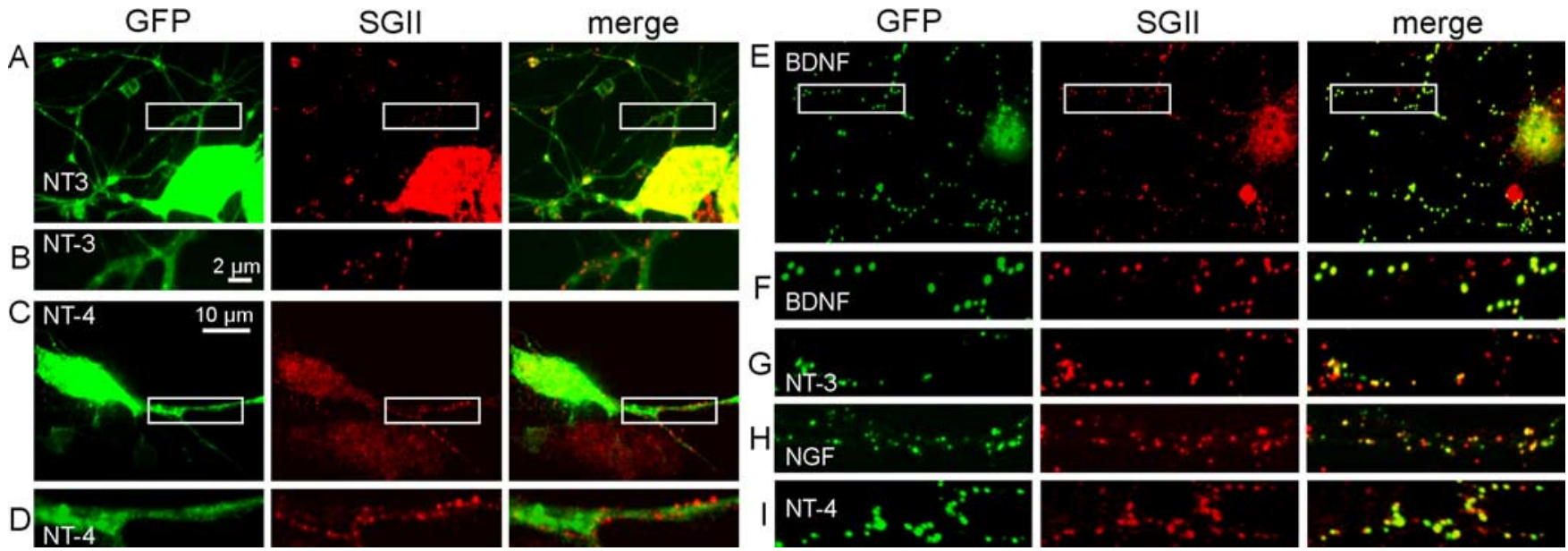

Figure 5. Colocalization of neurotrophins with secretogranin II. Hippocampal neurons were transfected at 8 DIV with the respective NTs and immunostained with an antibody directed against Sgll 2 d thereafter. In $A-I$, confocal images of GFP fluorescence signals (left), red fluorescent Sgll (middle), and a merged picture of both fluorescence channels (right) are shown for the same field of view. $\boldsymbol{A}-\boldsymbol{D}$, Typical examples of proximal expressers (here NT3-GFP and NT-4-GFP). White boxes in $\boldsymbol{A}$ and $\boldsymbol{C}$ are shown at higher magnification in $\boldsymbol{B}$ and $\boldsymbol{D}$. Note the absence of colocalization of NT signals with the secretory granule marker in dendrites, although these neurons clearly possess secretory granules in their dendrites. E-I, Colocalization of NTs with dendritic Sgll signals in distal expressers. E, BDNF-GFP-expressing neurons at low magnification. The boxed area is shown in $\boldsymbol{F}$ at higher magnification. $\mathbf{G}-\boldsymbol{I}$, Examples of Sgll colocalization of the other NTs at high magnification. Note the pronounced colocalization of dendritic NT signals with the secretory granule marker for all NTs in distal expressers.
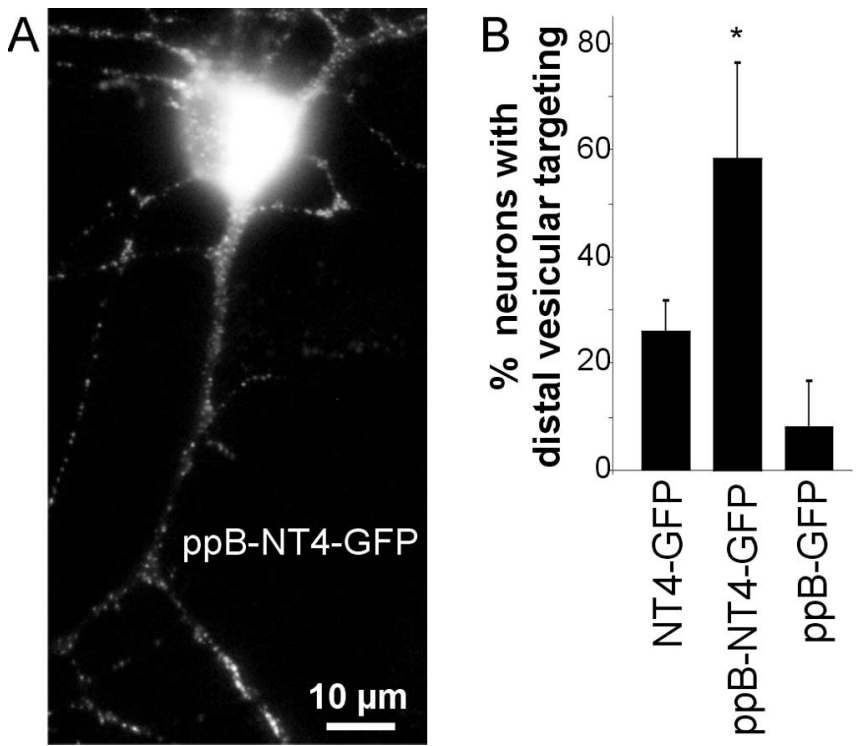

Figure 6. The pre-pro domain of BDNF redirects NT-4 to secretory granules. Hippocampal neurons were transfected at 8 DIV with the GFP-tagged NTs or NT domains as indicated and analyzed $2 \mathrm{~d}$ after transfection. $A$, Fusing the pre-pro domain of BDNF to the mature portion of NT-4 changed the localization pattern of NT-4, yielding more neurons with distal vesicular targeting. B, Quantification of targeting of the indicated proteins. ppB-GFP, Isolated pre-pro domain of BDNF tagged with GFP. *Significantly different from NT-4-GFP with $p<0.05$. Targeting of $\geq 20$ cells from $n \geq 3$ independent preparations was analyzed for each construct. Error bars represent SEs.

10 min of control superfusion, proximal expressers revealed a fluorescence decrease of $9.1 \pm 1.3 \%$ compared with $2.1 \pm 2.3 \%$ in distal expressers and $-0.3 \pm 1.6 \%$ in GFP controls (proximal expressers significantly different from distal expressers with $p<$ 0.02 and from GFP controls with $p<0.0005$ ). Thus, proximal (but not distal) expressers reveal a significant portion of the GFPtagged NT that is released via the constitutive pathway of secretion. Interestingly, subsequent depolarization of distal expressers revealed regulated secretion that was absent from proximal expressers (Fig. 10). These results indicate that the proximal expres- sion pattern reflects targeting of NTs to the constitutive pathway of secretion, whereas the distal vesicular expression pattern indicates efficient targeting to the regulated pathway of secretion.

Slow time course of synaptic secretion of neurotrophins The time course of synaptic NT release via the regulated pathway was further analyzed. On average (Fig. 11), the four NTs showed a loss of fluorescence intensity of $12-21 \%$ during 5 min stimulation with $50 \mathrm{mM} \mathrm{K}^{+}$. Because initial experiments with shorter imaging intervals (i.e., $300 \mathrm{~ms}$ ) failed to reveal any faster release component, the imaging interval was in all subsequent experiments matched to the observed release time course (i.e., $5 \mathrm{~s}$ interval). The fluorescence decrease could be fitted with a monoexponential function, yielding time constants of $121 \pm 29 \mathrm{~s}$ for NT-4 $(n=6$ cells $), 198 \pm 16 \mathrm{~s}$ for NGF $(n=4), 214 \pm 30 \mathrm{~s}$ for NT-3 $(n=8)$, and $307 \pm 78 \mathrm{~s}$ for BDNF $(n=5)$, respectively (NT-4 significantly different from NT-3 and BDNF with $p<0.05$ ). These results reveal a considerable heterogeneity in the speed of secretion of the different mammalian NTs and a faster release of NT-4 compared with the other NTs.

We used the lipophilic dye FM4-64 to determine the presynaptic release of conventional transmitters under identical stimulation conditions. The time course of FM destaining of presynaptic terminals has been shown previously to be a reliable relative measure of the average speed of transmitter vesicle exocytosis (Cochilla et al., 1999). As shown previously by others, high- $\mathrm{K}^{+}$induced release of FM4-64 in our experiments revealed an onset of secretion in $<5 \mathrm{~s}$, and release proceeded with a monoexponential decay time constant of $13 \pm 2 \mathrm{~s}(n=3$ cells) (Fig. $11 E)$, yielding a loss of $40 \%$ of the initial fluorescence intensity after $50 \mathrm{~s}$ of stimulation. Thus, the delay in onset and the time course of transmitter secretion is approximately fivefold to 10 -fold more rapid than the release of the fastest neurotrophin (NT-4, onset, $10-30 \mathrm{~s}, \tau=121 \pm 29 \mathrm{~s} ;$ FM4-64, onset, $<5 \mathrm{~s}, \tau=13 \pm 2 \mathrm{~s}$ ). To enable a direct comparison of the secretion time course regardless of the absolute amount of released substance, we normalized the average change in fluorescence intensity for FM4-64- and NTGFP-secreting cells, respectively (Fig. $11 F$ ). To exclude distortion of the release time course of BDNF and NT-3 by GFP unquench- 

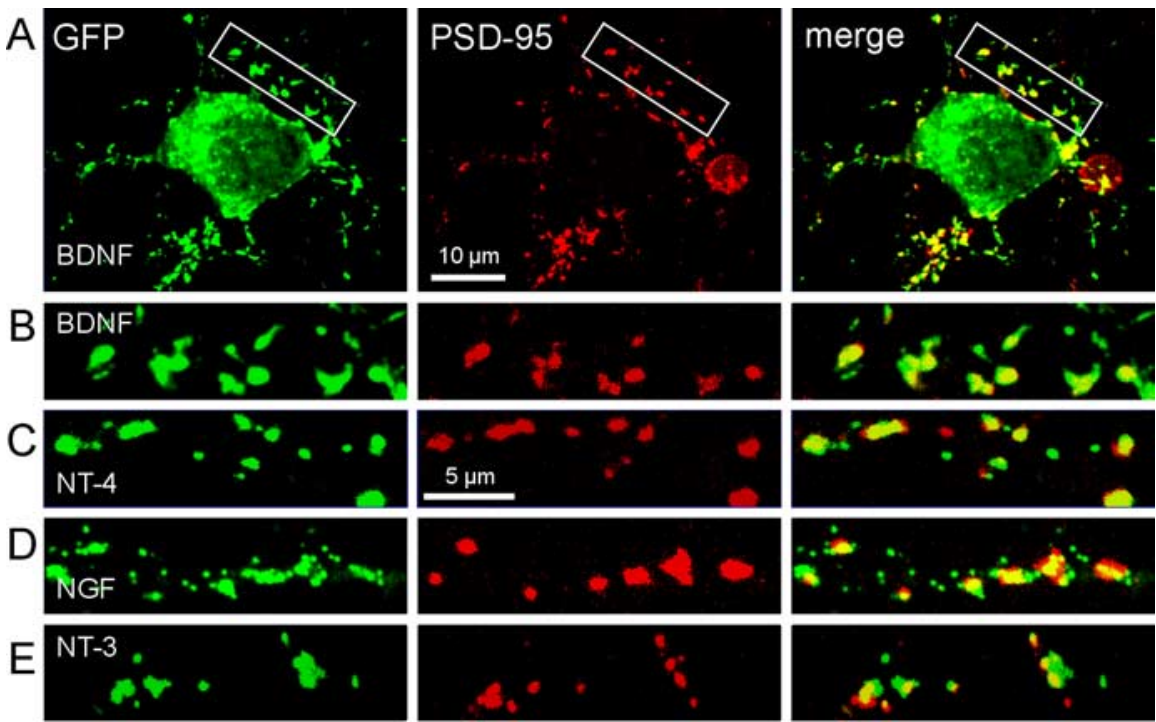

Figure 7. Colocalization of GFP-tagged NTs and PSD-95-DsRed after coexpression in the same cell. Hippocampal neurons were cotransfected with the respective GFP-tagged NT and PSD-95-DsRed (DNA ratio, 1:1) at 8 DIV, and cells were analyzed $2 \mathrm{~d}$ after transfection. Only cells with distal vesicular targeting are shown. In $\boldsymbol{A}-\boldsymbol{E}$, confocal images of GFP fluorescence signals (left), red fluorescent PSD-95 (middle), and a merged picture of both fluorescence channels (right) are shown for the same field of view. $\boldsymbol{A}$ BDNF-GFP-expressing neuron at low magnification. The white box is shown in $\boldsymbol{B}$ at higher magnification. $\boldsymbol{C}-\boldsymbol{E}$, Examples of PSD-95 colocalization of the other NTs at high magnification. Note the colocalization of NT vesicle clusters with PSD-95-DsRed, indicating targeting of all NTs to postsynaptic structures of glutamatergic synapses in distal vesicular expressers.
GFP
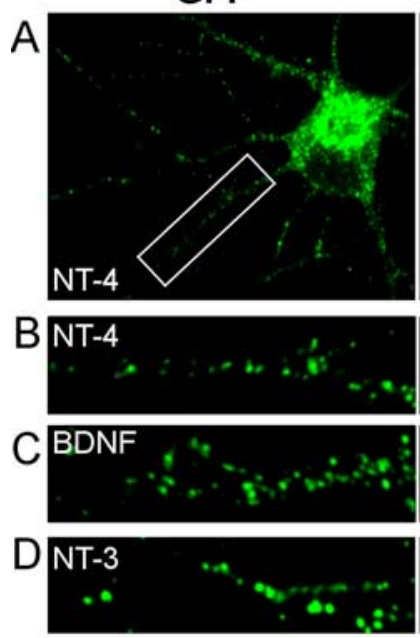

ENGF
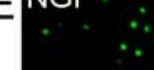
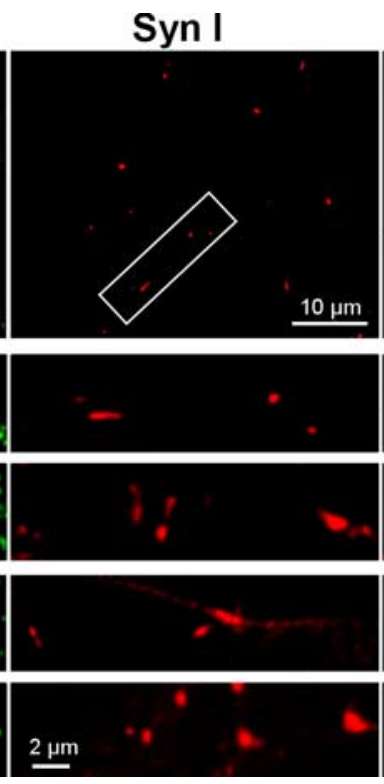

Figure 8. Neighboring localization of GFP-tagged NT vesicle clusters and the presynaptic marker synapsin I. Hippocampal neurons were transfected at 8 DIV with the respective NTs and fixed and immunostained with antibodies directed against the presynaptic marker protein Syn $2 \mathrm{~d}$ thereafter. Only cells with distal vesicular targeting are shown. In $\boldsymbol{A}-\boldsymbol{E}$, confocal images of GFP fluorescence signals (left), red fluorescent Synl (middle), and a merged picture of both fluorescence channels (right) are shown for the same field of view. $\boldsymbol{A}$, NT-4-GFP-expressing neuron at low magnification. The white box is shown in $\boldsymbol{B}$ at higher magnification. $C-E$, Examples of Synl staining for the other NTs at high magnification. Note the pronounced neighboring localization of the dendritic NT vesicle clusters and the presynaptic marker, indicating postsynaptic localization of all NTs. point of $50 \%$ fluorescence decrease), yielding $10 \pm 2$ s for FM4-64, compared with $75 \pm 12 \mathrm{~s}$ for NT-4 as the fastest neurotrophin and even higher values for the three other NTs (NT-3, $140 \pm 29$ s; NGF, $81 \pm 6 \mathrm{~s} ; \mathrm{BDNF}, 228 \pm 44 \mathrm{~s}$ ) (Fig. $11 \mathrm{~F}$, gray circles). This analysis suggests that secretion of the fastest NT proceeds at least seven times more slowly than transmitter release (half decay time; NT-4, $75 \pm 18 \mathrm{~s}$ vs FM4-64, $10 \pm 2$ s).

Peptide core solubilization has been shown previously to critically determine the speed of protein secretion from secretory granules, and neutralization of intragranular $\mathrm{pH}$ is a critical factor for peptide dissolution (Aspinwall et al., 1997; Han et al., 1999; Barg et al., 2002). We thus argued that the heterogeneity in the speed of NT secretion could reflect distinct degrees of $\mathrm{pH}$-dependent peptide condensation in the secretory granules containing the different NTs. To allow for similar rates of dissolution of all NTs, we thus preincubated our neurons with $4 \mu \mathrm{M}$ monensin, which is known to neutralize intragranular $\mathrm{pH}$ (Han et al., 1999). As is evident from Fig. 12 for BDNF-GFP, this treatment yielded the expected increase in fluorescence intensity, which was caused by $\mathrm{pH}$ dependent unquenching of GFP fluorescence. Similar changes in fluorescence were observed for the other NTs (compare Fig. 13). After reaching a steady-state level of fluorescence intensity, we provoked release by switching to $50 \mathrm{~mm} \mathrm{~K}{ }^{+}$solution. This resulted in a fast decrease of fluorescence intensity in the absence (15 of 15 cells) of any additional transient rise in fluorescence intensity. Importantly, under these conditions of prerelease neutralization of granules, all NTs revealed a nearly identical time course of secretion that was faster than release of NT-4 in the absence of monensin (onset, 10-20 s for all NTs; $\tau(\mathrm{BDNF})=81 \pm 22 \mathrm{~s} ; \tau(\mathrm{NGF})=76 \pm$ $29 \mathrm{~s} ; \tau(\mathrm{NT}-3)=92 \pm 23 \mathrm{~s} ; \tau(\mathrm{NT}-4)=$ $59 \pm 16 \mathrm{~s}$ ) (compare Figs. 11, 13). Also, the initial slope of the release time course (estimated from the half decay times) now approached similar values for all NTs (half decay; BDNF, $52 \pm 14 \mathrm{~s}$; NGF, $39 \pm 13 \mathrm{~s}$; NT-3, $51 \pm 13$ s; NT-4, $37 \pm 12$ s). However, this maximum speed of NT secretion that was accomplished by prerelease solubilization of NT cores was still four or five times slower than transmitter secretion as measured with FM4-64 (half decay time, ing in this analysis, only those cells were selected that did not show the initial fluorescence increase during depolarization (compare Fig. 9D). In this analysis, the initial slope of the fluorescence change, which is a measure of the probability of vesicle fusion, was approximated by the half decay time (i.e., the time
$10 \pm 2 \mathrm{~s}$ ). These data explain in part the reasons for the ten times slower release time course of NTs under physiological conditions (Fig. 11).

Overall, our data indicate that each NT, once it is targeted to synaptically localized secretory granules of glutamatergic syn- 
apses, can be released at these synapses with activity and with a slow time course that is typical for peptide secretion and that is incompatible with fast transmitterlike actions of NTs (cf. Kafitz et al., 1999). Furthermore, our data reveal that the speed of NT secretion is critically dependent on $\mathrm{pH}$-dependent dissolution of the NT cores inside the secretory granules.

\section{Discussion}

This is the first study to demonstrate synaptic secretion of NT-3, NT-4, and NGF from glutamatergic synapses. Using GFP-tagged NTs, we show targeting to granules of the regulated pathway of secretion and activitydependent synaptic release of all NTs from distal dendrites. However, whereas BDNF and NT-3 are destined for regulated synaptic secretion in the majority of neurons, NGF and NT-4 are in many cells rather targeted to the constitutive pathway. Thus, the likelihood to observe regulated synaptic secretion for the different NTs depends on the capacity of a given cell to localize the respective peptide to dendritic secretory granules. Once a NT reaches this compartment, activity-dependent synaptic secretion is warranted. Furthermore, the time course of synaptic secretion of all NTs is slower by a factor of 10 than conventional transmitter secretion.

\section{Intracellular localization of neurotrophins}

Endogenous BDNF was found to be released with activity in hippocampal and spinal cord preparations (Balkowiec and Katz, 2000, 2002; Lever et al., 2001). However, because of the low expression levels of endogenous BDNF, many details concerning BDNF localization were derived from BDNF- or BDNF-GFP-overexpressing CNS neurons. These studies indicated that BDNF is targeted to secretory granules in dendrites and axons of CNS neurons (Haubensak et al., 1998; Wu et al., 2004). Furthermore, BDNF vesicle clusters are found in the vicinity of active synapses and are released in response to synaptic stimulation (Hartmann et al., 2001; Kohara et al., 2001; Kojima et al., 2001). The intracellular fate and the sites of secretion of the other NTs, however, remained a highly controversial issue. Data from two laboratories suggested a similar intracellular targeting and release of all NTs (Heymach et al., 1996; Griesbeck et al., 1999; Wu et al., 2004). In contrast, NT-3, NT-4, and NGF were described by others to be released predominantly via the constitutive pathway (Mowla et al., 1999; Farhadi et al., 2000, Hibbert et al., 2003).

Our results now disclose that neuronal NT targeting can be better described as a cell-specific phenomenon, rather than allocating only constitutive or only regulated secretion to a given NT; BDNF and NT-3 show in a majority of neurons distal dendritic targeting of secretory granules, whereas NGF and NT-4 show more frequently the proximal expression pattern (Fig. 2). Our measurements of regulated and constitutive secretion (Figs. 9,
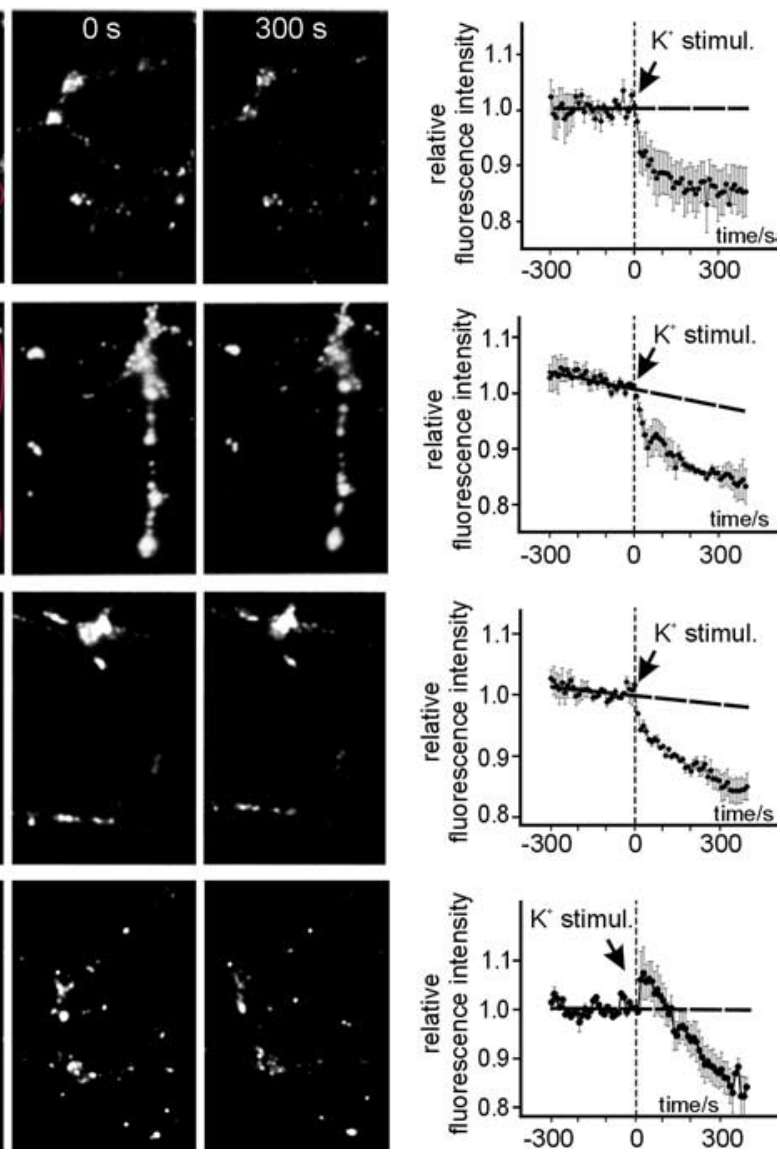

Figure 9. Synaptic secretion of all four mammalian NTs. Hippocampal neurons were cotransfected at 8 DIV with the respective GFP-tagged NT and PSD-95-DsRed ( $\boldsymbol{A}-\boldsymbol{C})$ or DsRed-VAMP $(\boldsymbol{D})$. At 10 DIV, dendritic NT vesicle clusters colocalizing with the secretion. In $\boldsymbol{A}-\boldsymbol{D}$, the picture on the left shows colocalization (yellow) of the synaptic marker (red) and the respective GFP-tagged

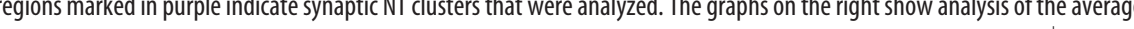
GF fluorescence intensity over time for the same cells as shown on the left. The arrows indicate the start of $50 \mathrm{~mm} \mathrm{~K}^{+}$-induced 作 and was often observed in cells expressing BDNF-GFP or NT-3-GFP (see Results). stimul., Stimulation.

10) allowed us to assign the proximal expression pattern being equivalent to targeting to the constitutive pathway, whereas the distal vesicular localization was identified to reflect targeting to the regulated pathway (Figs. 2, 5, 10). Thus, NGF and NT-4 are targeted to the regulated pathway in $23-46 \%$ of the cells, but the remaining neurons exhibit sorting of these NTs to the constitutive pathway. In contrast, NT-3 and especially BDNF are targeted almost exclusively to the regulated pathway of secretion. Thus, our data corroborate a targeting of NGF and NT-4 to the constitutive pathway in central neurons (Mowla et al., 1999; Hibbert et al., 2003). However, this was not an all-or-none phenomenon, and a considerable fraction of neurons displayed regulated secretion of NGF and NT-4 from distal dendritic granules (Figs. 2-9). The physiological relevance of this finding is stressed by our detection of distal vesicular expression (i.e., targeting to the regulated secretion pathway) of all endogenously expressed NTs (supplemental Fig. 2, available at www.jneurosci.org as supplemental material).

The recent controversial findings regarding NGF and NT-4 targeting (see above) could result from differences in brain areas 

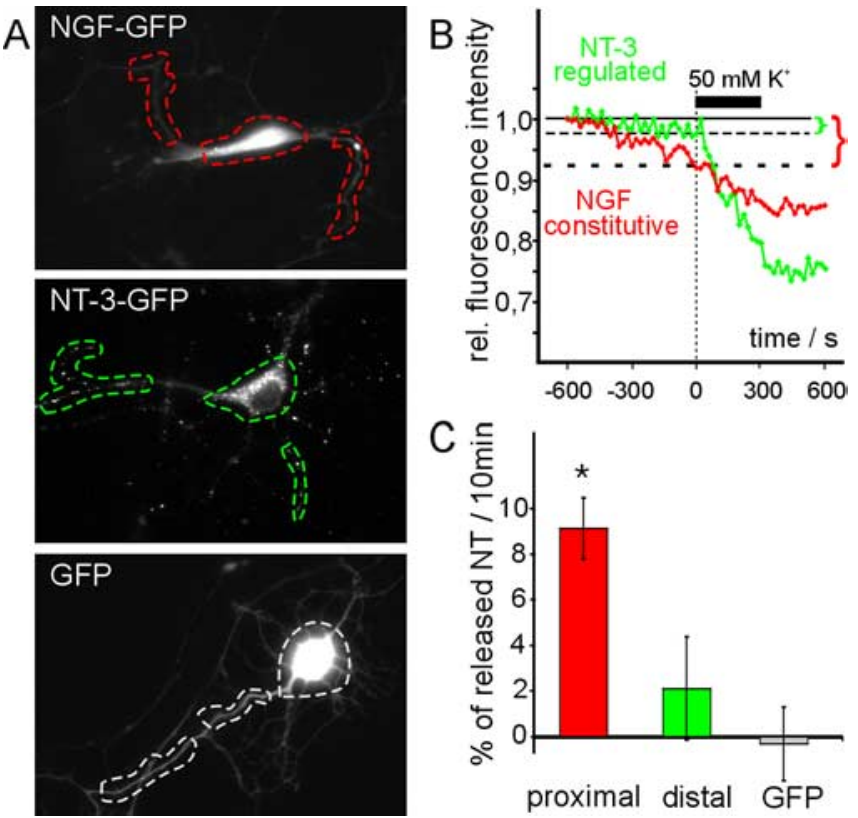

Figure 10. Proximal expressers show constitutive secretion. Transfected hippocampal neurons as in Figure 9. A, GFP fluorescence of a NGF-GFP proximal expresser, of a NT-3-GFP distal expresser, and of a GFP control. Marked regions were analyzed for changes in fluorescence intensity during superfusion with control solution $\left(0 \mathrm{~mm} \mathrm{Ca}{ }^{2+}, 1 \mu \mathrm{M} \mathrm{TTX).} \boldsymbol{B}\right.$, Time course of NT secretion of the two NT-expressing cells in $\boldsymbol{A}$ during $10 \mathrm{~min}$ control superfusion ( -600 to $0 \mathrm{~s}$ ) and subsequent depolarization with $50 \mathrm{~mm} \mathrm{~K}^{+}$in the presence of $2 \mathrm{~mm}$ extracellular $\mathrm{Ca}^{2+}$ (no TTX). Note the pronounced loss of intracellular NT fluorescence in the proximal expresser (NGFGFP; red bracket) and the absence of regulated secretion in this neuron. In contrast, the distal expresser (NT-3-GFP; green bracket) shows no comparable constitutive secretion but regulated release of the NT. rel., Relative. $\boldsymbol{C}$, Quantification of constitutive release as determined in $\boldsymbol{B}$ for proximal ( $n=15$ cells) and distal $(n=6)$ expressers. Proximal expressers show significantly more constitutive secretion than distal expressers $\left({ }^{*} p<0.02\right)$. Error bars represent SES.

and in the developmental status of neurons that were analyzed (see below). Interestingly, the coexistence of constitutive and regulated targeting of NGF in hippocampal neurons has been suggested previously (Blochl and Thoenen, 1996). Our results now reveal the varying prevalence of these two secretion pathways in different cells and that they are used differently by the four NTs.

We could switch a poorly distally targeted NT (NT-4-GFP) to become an effective distal localizer by simply swapping the prepro-BDNF domain to the mature portion of NT-4 (Fig. 6). This demonstrates the essential role of the pre-pro domains for the intracellular targeting of a given NT, while it provides a strong argument against a relevant role of the GFP tag for the targeting process. Furthermore, the undisturbed binding and subsequent activation of the respective Trk receptors by all GFP-tagged NTs in our study corroborates the physiological folding of all GFPtagged NTs (Fig. 1).

A large impact of the pre-pro domain of BDNF on its intracellular targeting has been suggested previously (Egan et al., 2003). However, a recent study by Lou et al. (2005) suggested an additional critical carboxypeptidase $\mathrm{E}$ consensus motif in the mature portion of BDNF, which is important for targeting of BDNF to the regulated pathway. Hence, future analysis should aim at clarifying the interplay between molecular cues in pro- versus mature NT domains in granular targeting. Furthermore, targeting and secretion routes for the different NTs might be governed by the developmental stage of neurons (Horton and Ehlers, 2003) and by cell-specific differences in targeting capacities (this study).

In proximal expressers, NTs are targeted to the ER, to the
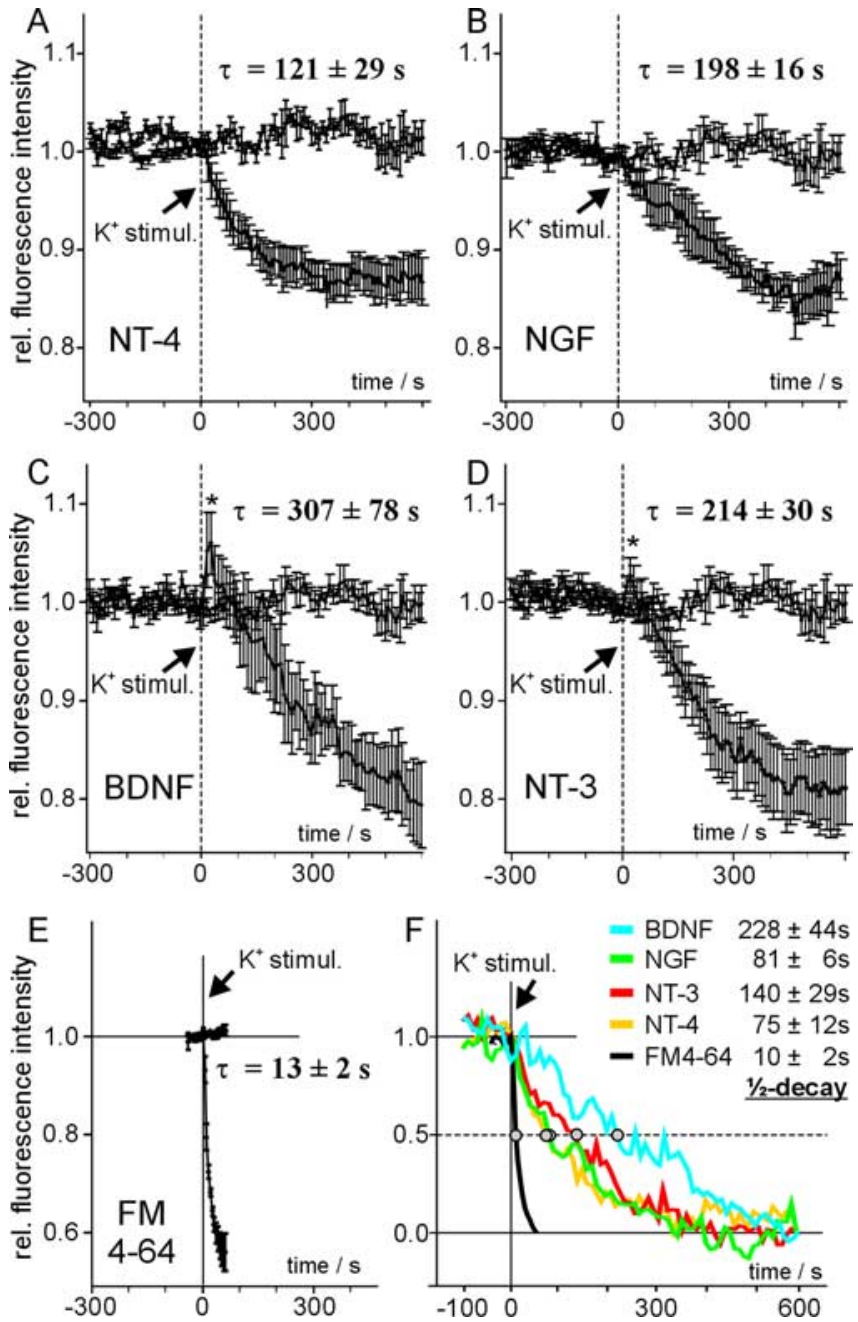

Figure 11. Differential time course of NT secretion in response to $\mathrm{K}^{+}$-induced depolarization. $\boldsymbol{A}-\boldsymbol{D}$, Averaged depolarization induced $\left(50 \mathrm{~mm} \mathrm{~K}^{+}\right)$fluorescence decrease for indicated NTs (NT-4, 6 cells; NGF, 4 cells; NT-3, 8 cells; BDNF, 5 cells). Error bars represent SEs. The vertical lines indicate the start of depolarization. The top curve in each graph represents averaged fluorescence intensity of negative controls ( $n=3$ cells; BDNF-GFP and NT-3-GFP expresser without $\mathrm{K}^{+}$-induced depolarization). Asterisks in $\mathbf{C}$ and $\boldsymbol{D}$ indicate fluorescence increase caused by GFP unquenching. $\boldsymbol{E}$, Average ( $n=3$ cells; $>50$ terminals per cell analyzed) time course of depolarization-induced $\left(50 \mathrm{~mm} \mathrm{~K}^{+}\right)$release of FM4-64-stained presynaptic terminals. Identical stimulation conditions as in $\boldsymbol{A} \mathbf{D}$. The top curve represents unstimulated negative controls. $\boldsymbol{F}$, Normalized fluorescence decrease of GFP-tagged NTs and FM4-64, respectively. Average over $n \geq 3$ cells for each condition. Only cells without initial GFP unquenching were selected. Note the different time points of half-maximal secretion (gray circles), representing the initial speed of compound release. rel., Relative; stimul., stimulation.

cis-Golgi and SgII-positive granules in the soma. These results are in agreement with previous studies showing widespread distribution of NGF and BDNF in the ER of hippocampal neurons (Blochl and Thoenen, 1996; Horton and Ehlers, 2003). Our study now relates the homogenous dendritic ER staining to targeting to the constitutive pathway, whereas in cells with targeting of a NT to the regulated pathway, the dendritic ER is no more a prominent site of NT storage (Fig. 3). This indicates that dendritic ER localization of NTs is detectable only if subsequent transportation to dendritic Golgi and secretory granules is hindered. Interestingly, NTs were also sorted to SgII-positive granules in the soma even of cells showing only constitutive secretion. This might reflect that NGF and NT-4 granules are generated in the soma but are retained there, whereas BDNF and NT-3 granules 

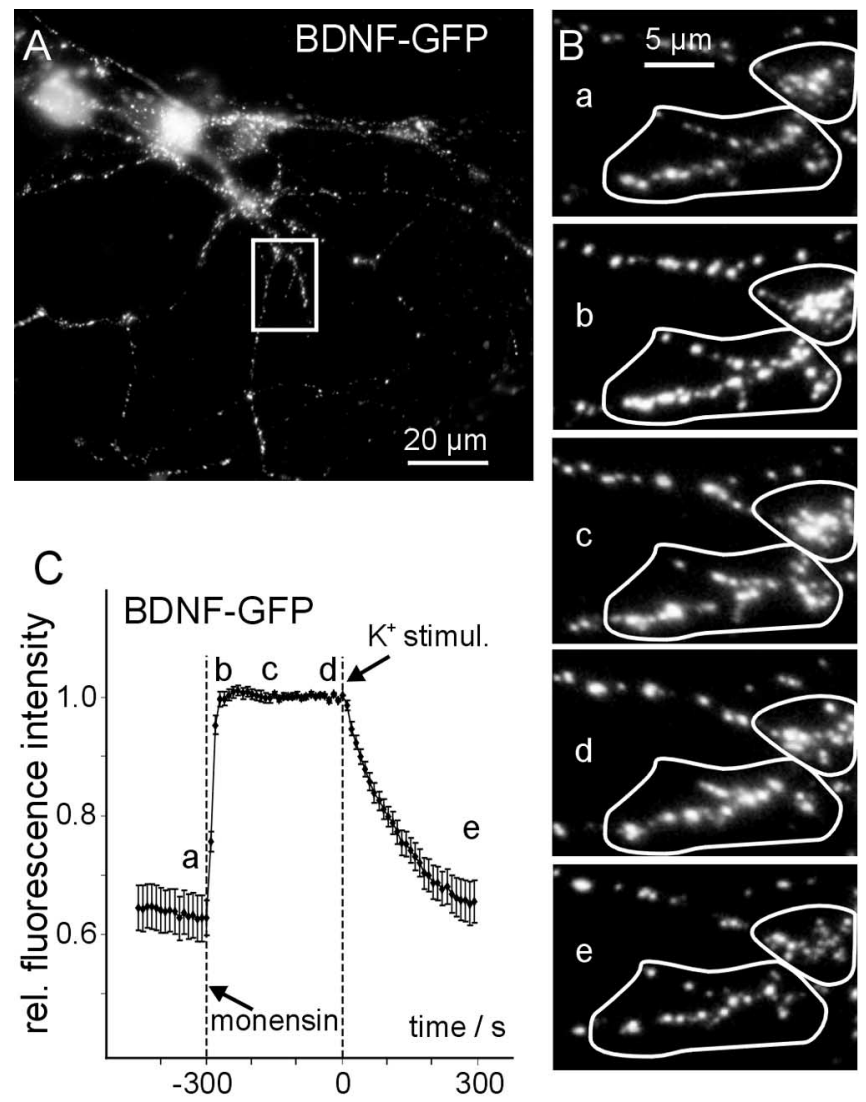

Figure 12. Neutralization of $\mathrm{pH}$ in secretory granules by monensin before depolarization speeds up the release of BDNF. Hippocampal neurons were transfected with GFP-tagged NTs at 8 DIV and used for secretion $2 \mathrm{~d}$ after transfection $\boldsymbol{A}, \mathrm{BDNF}-$ GFP-expressing neuron. $\boldsymbol{B}$, Time series of the marked box in $A$ at higher magnification. $C$, Analysis of fluorescence intensity of regions marked in $\boldsymbol{B}$. Note the pronounced increase in fluorescence intensity after application of monensin $(4 \mu \mathrm{m})$, which neutralizes pH in previously acidic granules. The letters a- e refer to the pictures shown in $\boldsymbol{B}$. Note the faster time course of BDNF secretion (compare Fig. 11) and the lack of GFP unquenching after $\mathrm{K}^{+}$-induced depolarization. rel., Relative; stimul., stimulation. Error bars represent SEs.

can efficiently enter the dendrites. Interestingly, also neurons showing sorting of a NT to the constitutive pathway clearly revealed secretory granules (albeit negative for the overexpressed NT) in dendrites (Fig. 5). This rules out that constitutive secretion was seen only because granules were generally absent from the dendrites of these neurons.

\section{Synaptic targeting and secretion of NTs}

Synaptic secretion of NGF, NT-3, and NT-4 has long remained elusive. Our study now provides evidence for activity-dependent synaptic secretion of these NTs, reminiscent of synaptic secretion of BDNF in hippocampal neurons (Hartmann et al., 2001). Judged by the prominent dendritic localization, the exact colocalization with the postsynaptic marker PSD-95 and the neighboring localization of the presynaptic markers SynI, VAMP, and FM4-64, the observed expression pattern speaks in favor of a dendritic postsynaptic localization of NTs at glutamatergic synapses. The efficiency of release is similar for all NTs, suggesting that the dendritic release machinery does not discriminate between vesicular cargoes. Thus, targeting of NTs to dendritic secretory granules warrants synaptic release via the regulated pathway.

Because BDNF is a sticky protein, the results of the BDNFGFP release assay could have been prolonged artificially by the washout kinetics of BDNF from the cell membrane. However, the
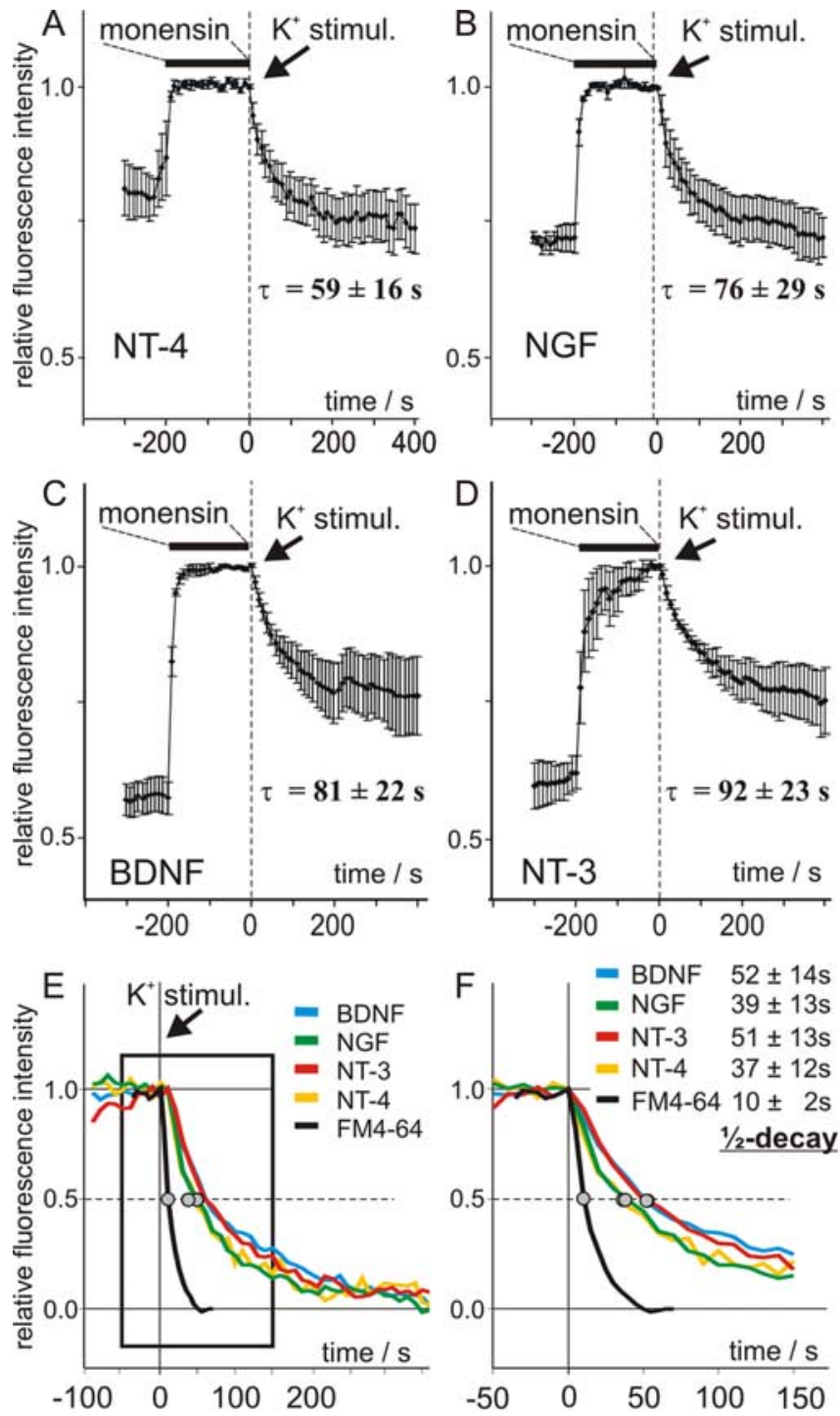

Figure 13. Average time course of NT secretion after neutralization of intragranular $\mathrm{pH}$ with monensin. $\boldsymbol{A}-\boldsymbol{D}$, Hippocampal neurons expressing the different GFP-tagged NTs were treated as in Figure 9. Monensin (4 $\mu \mathrm{m})$-induced fluorescence increase and acceleration of the time course of release during subsequent depolarization was observed for all NTs (compare Fig. 11). The $\tau$ values represent the results of monoexponential fit curves for $n \geq 4$ cells for each NT. $\boldsymbol{E}$, Normalized average depolarization induced $\left(50 \mathrm{~mm} \mathrm{~K}^{+}\right)$fluorescence decrease for indicated NTs after preincubation with monensin and for FM4-64 destaining of synaptic vesicles, respectively. The vertical line indicates start of depolarization. Average over $n \geq 4$ cells for each condition. $\boldsymbol{F}$, The boxed area in $\boldsymbol{E}$ at an expanded time scale. Note the similar half decay times (gray circles) for all NTs under these conditions but the still markedly faster speed of FM4-64 release from synaptic vesicles. Stimul., Stimulation. Error bars represent SEs.

exponential decay time constants for BDNF were not significantly different from those for NT-3 and NGF. More importantly, our results reveal that the release time course of these NTs is one order of magnitude more slowly than the release of FM464, which is a measure of transmitter secretion (Fig. 11). Thus, the slow release kinetics for NTs in our assay are not a specific feature of the sticky BDNF molecule but are valid also for NGF and NT-3. NT-4-GFP showed slightly faster release kinetics than the other three NTs. However, the NT-4 release time course was still seven times slower than transmitter secretion, matching the secretion speed of other modulatory peptides (Burke et al., 1997; Lang et al., 1997; Barg et al., 2002).

Because peptide secretion has been shown previously to de- 
pend critically on the speed of solubilization of peptide cores in secretory granules (Aspinwall et al., 1997), we argued that the faster release time course of NT-4 could reflect a higher degree of peptide condensation in vesicles containing BDNF, NGF, and NT-3. Indeed, neutralization of intragranular $\mathrm{pH}$ by monensin accelerated the release of the slow NTs to the value observed for NT-4. An effect of monensin on intragranular $\mathrm{pH}$ was evident from the fluorescence increase $30 \mathrm{~s}$ after the start of monensin application. Because high $\mathrm{K}^{+}$did not induce a further fluorescence increase, intragranular $\mathrm{pH}$ during monensin likely approached a similar neutralized value for all NTs. In addition, the monensin results support the notion that the transient $\mathrm{K}^{+}$induced fluorescence increase for BDNF and NT-3 under control conditions (Figs. 9, 11) was indeed a consequence of intragranular neutralization of granules in preparation for release, either by proton exchange via the initial fusion pore (Barg et al., 2002) or by proton efflux from the vesicles into the cytoplasm (Han et al., 1999).

The lack of an initial $\mathrm{K}^{+}$-induced brightening response in $\sim 50 \%$ of the BDNF-GFP- and NT-3-GFP-expressing cells under physiological conditions likely reflects neutralization of intragranular $\mathrm{pH}$ before the initiation of release. This suggests that secretory granules can exist in distinct prerelease states, differing in intragranular $\mathrm{pH}$. Interestingly, NT-4-GFP and NGF-GFP showed the initial brightening of GFP fluorescence in $<10 \%$ of the neurons, suggesting that their vesicles are neutralized more efficiently already before depolarization. This is in line with the smaller monensin-induced fluorescence increase for NGF and NT-4 compared with BDNF and NT-3 (Fig. 13).

Overall, these results suggest that intragranular $\mathrm{pH}$ and thus peptide solubilization could be the bottleneck for the speed of neurotrophin secretion, similar to observations for other peptides (Barg et al., 2002).

Judged by our peptide release assay that relies on diffusion limited washout of vesicular fluorescence, the NT release kinetics are slow in comparison to transmitter secretion monitored with a comparable diffusion limited assay (i.e., FM4-64 destaining). Importantly, given the lipophilic nature of FM dyes and the slow partitioning between lipid and aqueous phase of FM4-64 in particular, the FM4-64 washout measurements tend to underestimate the speed of transmitter release (Cochilla et al., 1999). In addition, the four times larger molecular weight of FM4-64 (MW, 600 daltons) compared with the small transmitter molecules (e.g., glutamate; MW, 147 daltons), and given that the diffusion coefficient is proportional to the root mean square of the molecular weight, diffusion speed of FM4-64 is $\sim 2.0$ times slower than diffusion of glutamate. This suggests that the kinetics of FM4-64 destaining tend to underestimate the true speed of glutamate release.

A similar calculation for GFP-tagged NTs yields a $\sim 1.8$ times slower diffusion speed of GFP-tagged versus untagged NTs (86 vs $28 \mathrm{kDa}$ ). Thus, our choice of fluorescent probes (i.e., FM4-64 and GFP-tagged NTs) is unlikely to have artificially expanded the relative differences in diffusion kinetics of transmitters versus NTs. Furthermore, based on this calculation, the diffusion time constant of glutamate is one order of magnitude bigger than that of NTs, being in line with the 5-10 times slower release time course of NTs in our study.

This also indicates that the rising phase of the NT concentration after provoking release is not steep enough to trigger rapid NT-evoked responses on a millisecond timescale (Kafitz et al., 1999; Blum et al., 2002).

\section{References}

Aspinwall CA, Brooks SA, Kennedy RT, Lakey JR (1997) Effects of intravesicular $\mathrm{H}+$ and extracellular $\mathrm{H}+$ and $\mathrm{Zn} 2+$ on insulin secretion in pancreatic beta cells. J Biol Chem 272:31308-31314.

Balkowiec A, Katz DM (2000) Activity-dependent release of endogenous brain-derived neurotrophic factor from primary sensory neurons detected by ELISA in situ. J Neurosci 20:7417-7423.

Balkowiec A, Katz DM (2002) Cellular mechanisms regulating activitydependent release of native brain-derived neurotrophic factor from hippocampal neurons. J Neurosci 22:10399-10407.

Barg S, Olofsson CS, Schriever-Abeln J, Wendt A, Gebre-Medhin S, Renstrom E, Rorsman P (2002) Delay between fusion pore opening and peptide release from large dense-core vesicles in neuroendocrine cells. Neuron 33:287-299.

Blochl A, Thoenen H (1995) Characterization of nerve growth factor (NGF) release from hippocampal neurons: evidence for a constitutive and an unconventional sodium-dependent regulated pathway. Eur J Neurosci 7:1220-1228.

Blochl A, Thoenen H (1996) Localization of cellular storage compartments and sites of constitutive and activity-dependent release of nerve growth factor (NGF) in primary cultures of hippocampal neurons. Mol Cell Neurosci 7:173-190.

Blum R, Kafitz KW, Konnerth A (2002) Neurotrophin-evoked depolarization requires the sodium channel $\mathrm{Na}(\mathrm{V}) 1.9$. Nature 419:687-693.

Boussif O, Lezoualc'h F, Zanta MA, Mergny MD, Scherman D, Demeneix B, Behr JP (1995) A versatile vector for gene and oligonucleotide transfer into cells in culture and in vivo: polyethylenimine. Proc Natl Acad Sci USA 92:7297-7301.

Burke NV, Han W, Li D, Takimoto K, Watkins SC, Levitan ES (1997) Neuronal peptide release is limited by secretory granule mobility. Neuron 19:1095-1102.

Cochilla AJ, Angleson JK, Betz WJ (1999) Monitoring secretory membrane with FM1-43 fluorescence. Annu Rev Neurosci 22:1-10.

Edwards RH, Selby MJ, Mobley WC, Weinrich SL, Hruby DE, Rutter WJ (1988) Processing and secretion of nerve growth factor: expression in mammalian cells with a vaccinia virus vector. Mol Cell Biol 8:2456-2464.

Egan MF, Kojima M, Callicott JH, Goldberg TE, Kolachana BS, Bertolino A, Zaitsev E, Gold B, Goldman D, Dean M, Lu B, Weinberger DR (2003) The BDNF val66met polymorphism affects activity-dependent secretion of BDNF and human memory and hippocampal function. Cell 112:257-269.

Ernfors P, Wetmore C, Olson L, Persson H (1990) Identification of cells in rat brain and peripheral tissues expressing mRNA for members of the nerve growth factor family. Neuron 5:511-526.

Ernfors P, Merlio JP, Persson H (1992) Cells expressing mRNA for neurotrophins and their receptors during embryonic rat development. Eur J Neurosci 4:1140-1158.

Farhadi HF, Mowla SJ, Petrecca K, Morris SJ, Seidah NG, Murphy RA (2000) Neurotrophin-3 sorts to the constitutive secretory pathway of hippocampal neurons and is diverted to the regulated secretory pathway by coexpression with brain-derived neurotrophic factor. J Neurosci 20:40594068.

Friedman WJ, Black IB, Kaplan DR (1998) Distribution of the neurotrophins brain-derived neurotrophic factor, neurotrophin-3, and neurotrophin-4/5 in the postnatal rat brain: an immunocytochemical study. Neuroscience 84:101-114.

Gauthier LR, Charrin BC, Borrell-Pages M, Dompierre JP, Rangone H, Cordelieres FP, De Mey J, MacDonald ME, Lessmann V, Humbert S, Saudou F (2004) Huntingtin controls neurotrophic support and survival of neurons by enhancing BDNF vesicular transport along microtubules. Cell 118:127-138.

Goodman LJ, Valverde J, Lim F, Geschwind MD, Federoff HJ, Geller AI, Hefti F (1996) Regulated release and polarized localization of brain-derived neurotrophic factor in hippocampal neurons. Mol Cell Neurosci 7:222-238.

Griesbeck O, Canossa M, Campana G, Gartner A, Hoener MC, Nawa H, Kolbeck R, Thoenen H (1999) Are there differences between the secretion characteristics of NGF and BDNF? Implications for the modulatory role of neurotrophins in activity-dependent neuronal plasticity. Microsc Res Tech 45:262-275.

Halban PA, Irminger JC (1994) Sorting and processing of secretory proteins. Biochem J 299:1-18. 
Han W, Li D, Stout AK, Takimoto K, Levitan ES (1999) $\mathrm{Ca}^{2+}$-induced deprotonation of peptide hormones inside secretory vesicles in preparation for release. J Neurosci 19:900-905.

Hartmann M, Heumann R, Lessmann V (2001) Synaptic secretion of BDNF after high-frequency stimulation of glutamatergic synapses. EMBO J 20:5887-5897.

Haubensak W, Narz F, Heumann R, Lessmann V (1998) BDNF-GFP containing secretory granules are localized in the vicinity of synaptic junctions of cultured cortical neurons. J Cell Sci 111:1483-1493.

Heese K, Inoue N, Nagai Y, Sawada T (2004) APP, NGF and the 'Sundaydriver' in a Trolley on the Road. Restor Neurol Neurosci 22:131-136.

Heymach Jr JV, Kruttgen A, Suter U, Shooter EM (1996) The regulated secretion and vectorial targeting of neurotrophins in neuroendocrine and epithelial cells. J Biol Chem 271:25430-25437.

Hibbert AP, Morris SJ, Seidah NG, Murphy RA (2003) Neurotrophin-4, alone or heterodimerized with brain-derived neurotrophic factor, is sorted to the constitutive secretory pathway. J Biol Chem 278:48129-48136.

Hofer M, Pagliusi SR, Hohn A, Leibrock J, Barde YA (1990) Regional distribution of brain-derived neurotrophic factor mRNA in the adult mouse brain. EMBO J 9:2459-2464.

Horton AC, Ehlers MD (2003) Dual modes of endoplasmic reticulum-toGolgi transport in dendrites revealed by live-cell imaging. J Neurosci 23:6188-6199.

Huttner WB, Gerdes HH, Rosa P (1991) The granin (chromogranin/secretogranin) family. Trends Biochem Sci 16:27-30.

Kafitz KW, Rose CR, Thoenen H, Konnerth A (1999) Neurotrophin-evoked rapid excitation through TrkB receptors. Nature 401:918-921.

Klau M, Hartmann M, Erdmann KS, Heumann R, Lessmann V (2001) Reduced number of functional glutamatergic synapses in hippocampal neurons overexpressing full-length TrkB receptors. J Neurosci Res 66:327336.

Kohara K, Kitamura A, Morishima M, Tsumoto T (2001) Activitydependent transfer of brain-derived neurotrophic factor to postsynaptic neurons. Science 291:2419-2423.

Kojima M, Takei N, Numakawa T, Ishikawa Y, Suzuki S, Matsumoto T, Katoh-Semba R, Nawa H, Hatanaka H (2001) Biological characterization and optical imaging of brain-derived neurotrophic factor-green fluorescent protein suggest an activity-dependent local release of brainderived neurotrophic factor in neurites of cultured hippocampal neurons. J Neurosci Res 64:1-10.

Korsching S, Auburger G, Heumann R, Scott J, Thoenen H (1985) Levels of nerve growth factor and its mRNA in the central nervous system of the rat correlate with cholinergic innervation. EMBO J 4:1389-1393.

Krijnse-Locker J, Parton RG, Fuller SD, Griffiths G, Dotti CG (1995) The organization of the endoplasmic reticulum and the intermediate compartment in cultured rat hippocampal neurons. Mol Biol Cell 6:13151332 .

Lang T, Wacker I, Steyer J, Kaether C, Wunderlich I, Soldati T, Gerdes HH, Almers W (1997) Ca2+-triggered peptide secretion in single cells imaged with green fluorescent protein and evanescent-wave microscopy. Neuron 18:857-863.

Lessmann V, Heumann R (1998) Modulation of unitary glutamatergic syn- apses by neurotrophin-4/5 or brain-derived neurotrophic factor in hippocampal microcultures: presynaptic enhancement depends on preestablished paired-pulse facilitation. Neuroscience 86:399-413.

Lessmann V, Gottmann K, Malcangio M (2003) Neurotrophin secretion: current facts and future prospects. Prog Neurobiol 69:341-374.

Lever IJ, Bradbury EJ, Cunningham JR, Adelson DW, Jones MG, McMahon SB, Marvizon JC, Malcangio M (2001) Brain-derived neurotrophic factor is released in the dorsal horn by distinctive patterns of afferent fiber stimulation. J Neurosci 21:4469-4477.

Lou H, Kim SK, Zaitsev E, Snell CR, Lu B, Loh YP (2005) Sorting and activity-dependent secretion of BDNF require interaction of a specific motif with the sorting receptor carboxypeptidase e. Neuron 45:245-255.

Lu B (2003) BDNF and activity-dependent synaptic modulation. Learn Mem 10:86-98.

Maisonpierre PC, Belluscio L, Friedman B, Alderson RF, Wiegand SJ, Furth ME, Lindsay RM, Yancopoulos GD (1990) NT-3, BDNF, and NGF in the developing rat nervous system: parallel as well as reciprocal patterns of expression. Neuron 5:501-509.

Mohrmann R, Lessmann V, Gottmann K (2003) Developmental maturation of synaptic vesicle cycling as a distinctive feature of central glutamatergic synapses. Neuroscience 117:7-18.

Mowla SJ, Pareek S, Farhadi HF, Petrecca K, Fawcett JP, Seidah NG, Morris SJ, Sossin WS, Murphy RA (1999) Differential sorting of nerve growth factor and brain-derived neurotrophic factor in hippocampal neurons. J Neurosci 19:2069-2080.

Nomoto H, Tomotoshi K, Ito H, Furukawa S (2000) Balance of two secretion pathways of nerve growth factor in PC12 cells changes during the progression of their differentiation, with a decrease in constitutive secretion in more differentiated cells. J Neurosci Res 59:632-642.

Ozawa H, Takata K (1995) The granin family-its role in sorting and secretory granule formation. Cell Struct Funct 20:415-420.

Poo MM (2001) Neurotrophins as synaptic modulators. Nat Rev Neurosci $2: 24-32$.

Sambrook J, Fritsch EF, Maniatis T (1989) Molecular cloning-a laboratory manual, Ed 3. Cold Spring Harbor, NY: Cold Spring Harbor Laboratory.

Swanwick CC, Harrison MB, Kapur J (2004) Synaptic and extrasynaptic localization of brain-derived neurotrophic factor and the tyrosine kinase $B$ receptor in cultured hippocampal neurons. J Comp Neurol 478:405-417.

Tischler AS, Greene LA (1978) Morphologic and cytochemical properties of a clonal line of rat adrenal pheochromocytoma cells which respond to nerve growth factor. Lab Invest 39:77-89.

Weclewicz K, Svensson L, Kristensson K (1998) Targeting of endoplasmic reticulum-associated proteins to axons and dendrites in rotavirusinfected neurons. Brain Res Bull 46:353-360.

Wu YJ, Kruttgen A, Moller JC, Shine D, Chan JR, Shooter EM, Cosgaya JM (2004) Nerve growth factor, brain-derived neurotrophic factor, and neurotrophin-3 are sorted to dense-core vesicles and released via the regulated pathway in primary rat cortical neurons. J Neurosci Res 75:825-834.

Zhou XF, Rush RA (1994) Localization of neurotrophin-3-like immunoreactivity in the rat central nervous system. Brain Res 643:162-172. 